\title{
INTERCOMPARISON EXPERIMENTS ON DISSOLVED TRACE- METAL DATA FROM THE MISSISSIPPI RIVER AND SOME OF ITS
}

TRIBUTARIES, 1989-90

by H.E. Taylor, A.M. Shiller, J.R. Garbarino, and T.I. Brinton

U.S. GEOLOGICAL SURVEY

Open-File Report 93-628 


\section{U.S. DEPARTMENT OF THE INTERIOR \\ BRUCE BABBITT, Secretary \\ U.S. GEOLOGICAL SURVEY \\ Gordon P. Eaton, Director}

The use of trade, product, industry, or firm names is for descriptive purposes only and does not imply endorsement by the U.S. Government.

For additional information write to:

Chief, Branch of Regional Research

U.S. Geological Survey

Box 25046, MS 418

Denver Federal Center

Denver, CO 80225
Copies of this report can be purchased from:

U.S. Geological Survey

Earth Science Information Center

Open-File Reports Section

Box 25286, MS 517

Denver Federal Center

Denver, CO 80225 


\section{CONTENTS}

Abstract

Introduction

Purpose and scope

Acknowledgments.

Pilot study

Data collection, sample processing, and chemical-analysis protocols..

Comparison of trace-metal data

References cited

\section{FIGURES}

Figure 1. Map showing Mississippi River, tributaries, and location of sampling sites

2. Diagram showing sample collection, processing, and chemical-analysis protocols for June 1989 pilot comparison study in the Mississippi River

3. Diagram showing sample collection, processing, and chemical analysis protocols for June 1990 comparison study in the Mississippi River and some of its tributaries

4-10. Graphs showing relation between dissolved:

4. Aluminum concentrations in samples collected and processed by U.S. Geological Survey National Stream Quality Accounting Network Program (NASQAN) and National Research Program (NRP), June 1990.

5. Cadmium concentrations in samples collected and processed by U.S. Geological Survey National Stream Quality Accounting Network Program (NASQAN) and National

Research Program (NRP), June 1990.

6. Chromium concentrations in samples collected and processed by U.S. Geological Survey National Stream Quality Accounting Network Program (NASQAN) and National Research Program (NRP), June 1990

7. Copper concentrations in samples collected and processed by U.S. Geological Survey National Stream Quality Accounting Network Program (NASQAN) and National Research Program (NRP), June 1990

8. Lead concentrations in samples collected and processed by U.S. Geological Survey National Stream Quality Accounting Network Program (NASQAN) and National Research Program (NRP), June 1990

9. Zinc concentrations in samples collected and processed by U.S. Geological Survey National Stream Quality Accounting Network Program (NASQAN) and National Research Program (NRP), June 1990

10. Iron concentrations in samples collected and processed by U.S. Geological Survey National Stream Quality Accounting Network Program (NASQAN) and National Research Program (NRP) (analysis by Branch of Analytical Services), June 1990 


\section{TABLES}

1. Summary of selected trace-metal concentrations in the Mississippi River near St. Francisville, Louisiana, June 26, 1989

2. Summary of selected trace-metal concentrations in the Mississippi River below Belle Chasse, Louisiana, June 28, 1989

3. U.S. Geological Survey National Stream Quality Accounting Network sampling sites with corresponding

District offices

Tables 4-13. Summary of trace-metal concentrations in the:

4. Illinois River at Valley City, Illinois, June 7, 1990

10

5. Mississippi River below Grafton, Illinois, June 11, 1990

6. Mississippi River at Thebes, Illinois, June 13, 1990

7. Ohio River at Olmsted, Illinois, June 14, 1990 13

8. Mississippi River below Memphis, Tennessee, June 18, 1990 14

9. Mississippi River below Arkansas City, Arkansas, June 20, 1990 15

10. Yazoo River below Steele Bayou, Mississippi, June 22, 1990 16

11. Mississippi River below Vicksburg, Mississippi, June 23, 1990

12. Mississippi River near St. Francisville, Louisiana, June 25, 1990

13. Mississippi River below Belle Chasse, Louisiana, June 27, 1990 


\begin{tabular}{rll}
\hline Multiply & By & To obtain \\
\hline milliliter $(\mathrm{mL})$ & Volume & \\
liter $(\mathrm{L})$ & 0.03382 & ounces, fluid \\
& 0.2642 & gallon \\
& & \\
picogram $(\mathrm{pg})$ & Mass & \\
microgram $(\mu \mathrm{g})$ & 0.00000000003257 & ounce, avoirdupois \\
milligram $(\mathrm{mg})$ & 0.00000003527 & ounce, avoirdupois \\
gram $(\mathrm{g})$ & 0.00003527 & ounce, avoirdupois \\
& 0.03527 & ounce, avoirdupois \\
& & \\
millimeter $(\mathrm{mm})$ & Length & inches \\
micrometer $(\mu \mathrm{m})$ & 25.4 & inches \\
& 0.0254 & \\
\hline
\end{tabular}

Sea level: In this report "sea level" refers to the National Geodetic Vertical Datum of 1929 (NGVD of 1929)-a geodetic datum derived from a general adjustment of the first-order level nets of both the United States and Canada, formerly called Sea Level Datum of 1929. 


\title{
INTERCOMPARISON EXPERIMENTS ON DISSOLVED TRACE-METAL DATA FROM THE MISSISSIPPI RIVER AND SOME OF ITS TRIBUTARIES, 1989-90
}

\author{
By H.E. Taylor, A.M. Shiller, J.R. Garbarino, and T.I. Brinton
}

\begin{abstract}
A series of experiments was performed at several U.S. Geological Survey National Stream Quality Accounting Network (NASQAN) sampling sites on the Mississippi River and selected tributaries to directly compare and identify differences in dissolved trace-metal quantitation using NASQAN protocols and National Research Program (NRP) sampling and analysis procedures. NASQAN samples were collected using discharge-weighted, depth and width-integrated sampling procedures; field processing and laboratory analyses followed standard protocols that do not include ultraclean techniques. Samples were collected by NRP personnel using discharge-weighted, depthand width-integrated sampling procedures. NRP samples were processed by ultraclean handling techniques and low-concentration-level determinations of $\mathrm{Al}, \mathrm{As}, \mathrm{B}, \mathrm{Ba}, \mathrm{Be}, \mathrm{Ca}, \mathrm{Cd}, \mathrm{Co}, \mathrm{Cr}, \mathrm{Cu}, \mathrm{Li}$, $\mathrm{Mg}, \mathrm{Mn}, \mathrm{Mo}, \mathrm{Na}, \mathrm{Pb}, \mathrm{Se}, \mathrm{SiO}_{2}, \mathrm{Sr}, \mathrm{Tl}, \mathrm{U}, \mathrm{V}$, and $\mathrm{Zn}$ were performed by inductively coupled plasmamass and inductively coupled plasma-atomic emission spectrometric methods. Comparable samples were also processed and analyzed by the University of Southern Mississippi for $\mathrm{Al}, \mathrm{Cd}, \mathrm{Cu}, \mathrm{Fe}, \mathrm{Mo}$, $\mathrm{Ni}, \mathrm{V}$ and $\mathrm{Zn}$ by electrothermal-vaporization atomic absorption spectrometric methods preceded by a coprecipitation preconcentration technique.
\end{abstract}

Data from these comparative experiments are tabulated in this report. Figures including data separating sampling and sample-processing effects are also presented.

\section{INTRODUCTION}

Water samples form the U.S. Geological Survey (USGS) National Stream Quality Accounting Network (NASQAN) have routinely been collected and analyzed on a monthly basis from 1973 to 1980 at as many as 500 sites located throughout the United States. Since 1980, the sampling frequency has decreased, so that presently, 42 percent of stations are sampled quarterly and 58 percent bimonthly. This network, along with the U.S. Geological Survey National Stream Quality Surveillance System (NWQSS), which was operated from 1976 to 1981, has been the basis of a comprehensive monitoring program to establish long-term trends of water quality in the Nation's streams and rivers (Briggs and Ficke, 1978). Common constituents including major cations and anions, nutrients, alkalinity, $\mathrm{pH}$, dissolved oxygen, bacteria, and suspended sediment have been measured on a monthly schedule. Selected trace metals have been analyzed in specially preserved samples on a quarterly basis. Sampling and analysis protocols have followed the guidelines established and reported by Smith and others (1987).

Collection of samples and field processing are carried out by many different persons, depending on the specific USGS District or Subdistrict program area in which the sampling site is located. In addition, each USGS sampling team has its own set of sampling and sample-processing equipment, which can be maintained and used in a variable fashion. All samples are shipped to and analyzed by the U.S. Geological Survey, Branch of Analytical Services (BAS) in Arvada, Colorado, using approved published methodology (Skougstad and others, 1979). 


\section{Purpose and Scope}

The purpose of this report is to tabulate low-concentration, dissolved trace-element data obtained from an intercomparison experiment, jointly performed by the U.S. Geological Survey's National Research Program (NRP) and the University of Southern Mississippi. These experiments were designed to assist in identifying the source and nature of discrepancies in data from the NASQAN program (Shiller and Boyle; Windom and others, 1991).

Since 1987, the National Research Program (NRP) of the USGS has conducted a comprehensive research study on the occurrence, distribution, transport and processes affecting dissolved contaminants and contaminants associated with suspended material in the Mississippi River and some of its tributaries. As part of that study, depth-integrated sampling techniques for collecting water and suspended material (originally designed by Nordin and others, 1983; Meade and others, 1985; and Meade, 1985) have been developed further to include procedures for separating suspended materials, differentiating size fractions, and for preservation and transport of samples to the laboratory for chemical analysis (Leenheer and others, 1989; Meade and Stevens, 1990). In addition, specialized analytical laboratory methods have been developed to permit accurate and precise measurement of the concentration of specific constituents in the samples. These analytical techniques include those optimized for the measurement of trace organic compounds, especially agrochemicals (Pereira and others, 1990) and those developed for the semiquantitative and quantitative determination of trace metals (Taylor, 1989; Taylor and Garbarino, 1991).

By the end of 1990 , this study had consisted of seven sampling cruises utilizing the research vessel ACADIANA on the Mississippi River and some of its tributaries. Samples were collected and analyzed at as many as 20 preselected sites along the river under a variety of hydrologic conditions. Results of the measurement of trace metals during the first three sampling cruises (July-August 1987, November-December 1987 and May-June 1988) are summarized by Taylor and others (1990). Hydrologic and sedimentologic data for the July-August 1987, NovemberDecember 1987, and May-June 1988 sampling cruises are given by Moody and Meade (1992) and for the June 1989 and June 1990 sampling cruises by Moody and Meade (1993). 


\section{Acknowledgments}

Special thanks are given to David Roth for his assistance in completing the laboratory analyses and compiling data for this report. Thanks are also expressed to J. F. Williamson for her assistance in compiling the National Water Quality Laboratory data. Gratitude is expressed to the many colleagues who assisted in collecting samples and providing field logistics including: E.V. Axtmann, Ian Droppo, Daphne Frilot, J.A. Leenheer, R.H. Meade, T.L. Miller, J.A. Moody, T.I. Noyes, Jr., T.F. Rees, C.E. Rostad, J.L. Seeley, R. Stallard, H.H. Stevens, Jr., and the ACADIANA crew, Wilton Delaune, Chuck Guidry, Craig LeBoeuf, and Wayne Simoneaux.

District NASQAN samples were collected in the field by the following people:

Illinois District: A.L. Bewsher, G.K. Boughton and P.D. Hayes.

Missouri District: J.V. Davis, S.R. Femmer, D. Hatten, and W. Mills.

Kentucky District: A.C. Brown and G.L. Hubbard.

Arkansas District: C.K. Heavener, J.A. Jones and E.E. Morris.

Mississippi District: P. Grantham, F. Morris and L.J. Slack.

Louisiana District: V.G. Bergeron, C.R. Demas, K.L. Johnson, T. Kolb,

C.K. Labbe, M.L. Ross and S. Woodley.

\section{PILOT STUDY}

During the research cruise of June 1989, a pilot study was performed to compare data collected according to NASQAN sampling, handling and analysis procedures with those collected according to NRP research methods. Two sampling sites were involved-Mississippi River near

St. Francisville, La., and Mississippi River below Belle Chasse, La. (see fig. 1). In the pilot study, samples were collected at the same time and location by both the NRP project personnel and NASQAN project personnel. The NRP project personnel collected both a surface grab sample, similar in nature to those previously collected by Shiller and Boyle $(1983,1987)$, and a dischargeweighted sample which is referred to hereinafter as a depth-integrated sample (Meade and Stevens, 1990). The NASQAN personnel collected a sample using the standard NASQAN protocol, which specifies a depth-integrated sample. Statistically representative splits of these samples (except the surface grab) were made both before and after the field filtration process to remove suspended matter. The sample splits were exchanged between groups, and unfiltered portions were also processed using the other group's procedure.

The NRP filtration process for both the depth- and width-integrated and the surface grab sample consisted of exhaustive vacuum filtration of approximately $250 \mathrm{~mL}$ of raw sample through a 47-mm-diameter, $0.4-\mu \mathrm{m}$ pore size Nuclepore filter, using an all-Teflon filter-holder assembly. This filtration was accomplished in such a way as to effectively clog the pores of the filter with the suspended matter from the initial portion of sample passed through the membrane; therefore, the exact particle-size cutoff cannot be precisely defined. Filters and apparatus were cleaned prior to sample filtration by rinsing with $50 \mathrm{~mL}$ of 0.1 -percent $\mathrm{HNO}_{3}$ (quartz distilled; Kuehner and others, 1972), $50 \mathrm{~mL}$ of 18-megohm deionized $\mathrm{H}_{2} \mathrm{O}$, and finally rinsed with an aliquot of sample prior to final filtration. Filtered samples were acidified with $1 \mathrm{~mL}$ of concentrated $\mathrm{HNO}_{3}$ per $250 \mathrm{~mL}$ of filtered sample. The NASQAN personnel processed the samples by the standard NASQAN protocols, which require passing $200 \mathrm{~mL}$ of raw sample through a $142-\mathrm{mm}$-diameter, $0.45-\mu \mathrm{m}$ poresize membrane filter using an all-plastic filter-holder assembly prior to collection of sample filtrate for chemical analysis.

This resulted in a set of samples (see fig. 2) that consisted of the following: (a) sample collected and filtered by NRP; (b) sample collected by NRP and filtered by NASQAN personnel; (c) sample 


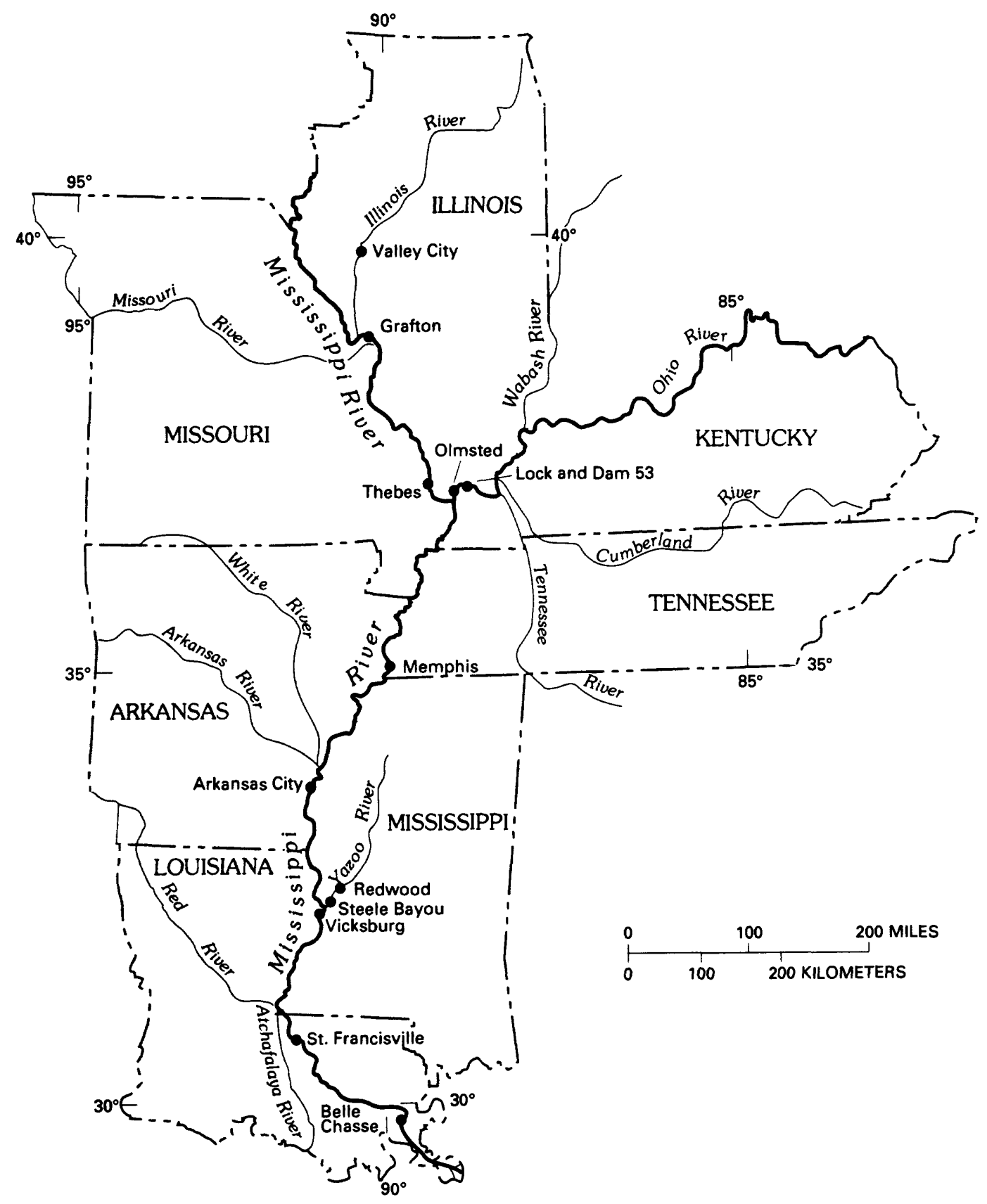

Figure 1.--Mississippi River, tributaries, and location of sampling sites. 

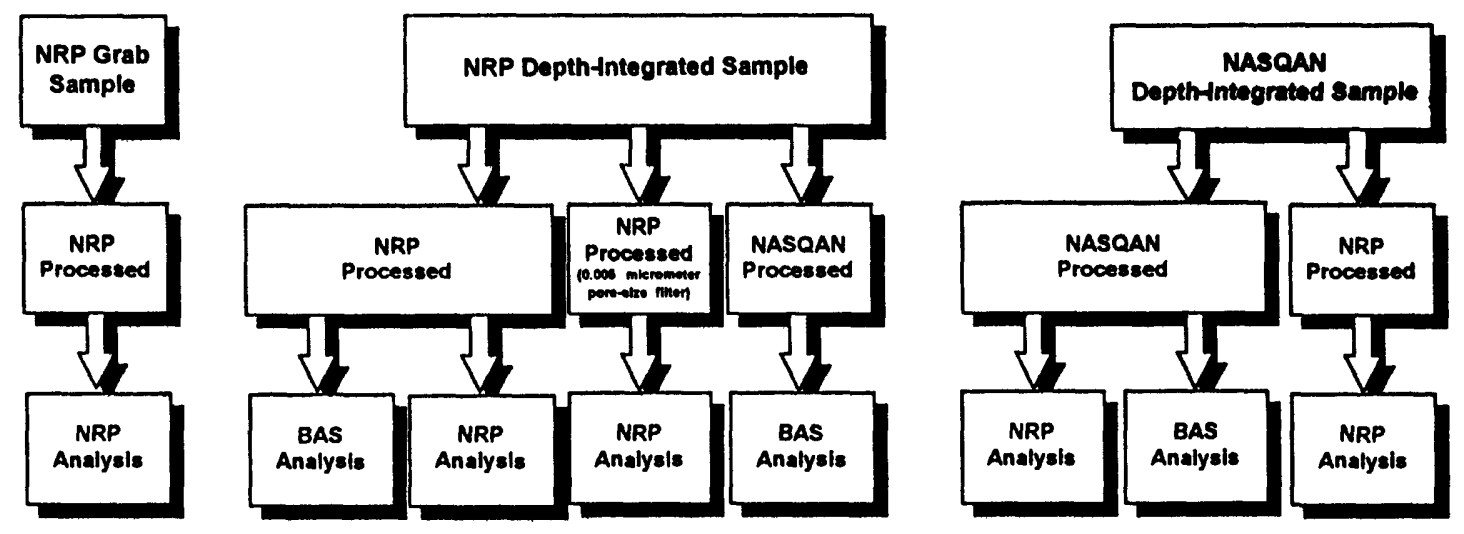

Figure 2.--Sample collection, processing, and chemical-analysis protocols for June 1989 pilot comparison study in the Mississippi River. NRP, NASQAN, and BAS represent U.S. Geological Survey National Research Program, National Stream Quality Accounting Network, and Branch of Analytical Services, respectively.

collected and filtered by the NASQAN personnel; and (d) sample collected by NASQAN personnel and filtered by NRP personnel. In addition to these samples and the previously mentioned surface grab sample, the remainder of the NRP depth-integrated sample was bulk processed as described by Leenheer and others (1989), resulting in a filtrate sample in which all particulate matter having a particle diameter greater than $0.005 \mu \mathrm{m}$ had been removed.

Sample splits were submitted to the BAS for standard NASQAN sample analysis, and sub-splits were analyzed by the BAS's Research and Methods Development group. The NRP sample splits were analyzed by custom-developed inductively coupled plasma-atomic emission spectrometric (Garbarino and Taylor, 1979 and 1980) and inductively coupled plasma-mass spectrometric techniques (Taylor and others, 1990; Taylor and Garbarino, 1991).

The results of this experiment are summarized in tables 1 and 2, for the Mississippi River below Belle Chasse, La., and Mississippi River near St. Francisville, La., sites, respectively. Generally, excellent agreement (percent differences from 10 to 50, depending on concentration level) is observed for most trace elements between the respective samples. However, those elements with the lowest concentrations (including, $\mathrm{Fe}, \mathrm{Cu}$, and $\mathrm{Zn}$ ) might show some anomalous characteristics that could be explained by one or more effects, including contamination, filtration, preservation, and sensitivity of the analytical techniques used. A more detailed analysis of this data set with particular attention to precision could result in improved interpretation of past and future network data sets. Results of this experiment encouraged a more widespread and extensive study involving other NASQAN project offices and sampling locations with the goal being to use the consistent NRP sampling and analysis protocols to "calibrate" the measurement of dissolved trace metals at a variety of sampling sites under a variety of hydrologic and sampling conditions (personnel, equipment, and techniques). This permitted an assessment to be made of the overall precision and consistency of data collected in the network monitoring programs and identify and evaluate specific sampling and handling problems, such as sampling equipment malfunction and contamination. 
Table 1.-Summary of selected trace-metal concentrations in the Mississippi River near St. Francisville, Louisiana, June 26, 1989

[Samples collected, processed, and analyzed by U.S. Geological Survey National Stream Quality Accounting Network (NASQAN), National Research Program (NRP), and Branch of Analytical Services (BAS). All constituents reported in micrograms per liter, except $\mathrm{Ca}, \mathrm{Mg}, \mathrm{Na}$, and $\mathrm{SiO}_{2}$, which are reported in milligrams per liter]

\begin{tabular}{|c|c|c|c|c|c|c|c|c|}
\hline \multirow{3}{*}{$\begin{array}{c}\begin{array}{c}\text { Sample } \\
\text { collection } \\
\text { protocol }\end{array} \\
\text { Sample } \\
\text { process } \\
\text { protocol }\end{array}$} & \multirow{3}{*}{$\begin{array}{l}\text { NRP } \\
\text { grab } \\
\text { NRP } \\
\text { NRP }\end{array}$} & \multicolumn{4}{|c|}{ NRP depth-Integrated } & \multicolumn{3}{|c|}{ NASQAN depth-Integrated } \\
\hline & & \multicolumn{2}{|c|}{ NRP } & \multirow{2}{*}{$\begin{array}{c}\text { NRP1 } \\
\text { NRP }\end{array}$} & \multirow{2}{*}{$\begin{array}{c}\text { NASQAN } \\
\text { BAS }\end{array}$} & \multirow{2}{*}{$\begin{array}{c}\text { NASQAN } \\
\text { NRP }\end{array}$} & \multirow{2}{*}{$\begin{array}{c}\text { NASQAN } \\
\text { BAS }\end{array}$} & \multirow{2}{*}{$\begin{array}{c}\text { NRP } \\
\text { NRP }\end{array}$} \\
\hline & & BAS & NRP & & & & & \\
\hline \multicolumn{9}{|l|}{ Constituent } \\
\hline $\mathbf{B a}$ & 52.2 & 48 & 49.3 & 49.5 & 48 & 47.9 & 49 & 48.9 \\
\hline $\mathrm{Be}$ & $<.02$ & $<.5$ & $<.02$ & $<.02$ & $<.5$ & .03 & $<.5$ & .04 \\
\hline $\mathrm{Ca}$ & 32.7 & 32 & 32.1 & 32.2 & 32 & 32.3 & 32 & 31.9 \\
\hline Cd & $<.1$ & $<.1$ & $<.1$ & $<.1$ & .2 & .2 & .2 & 0.4 \\
\hline Co & $<.03$ & $<.1$ & $<.03$ & $<.03$ & $<.1$ & .1 & $<.1$ & .06 \\
\hline $\mathbf{C r}$ & $<.2$ & $<5$ & $<.2$ & $<.2$ & $<5$ & $<.2$ & $<5$ & $<.2$ \\
\hline $\mathrm{Cu}$ & 2.2 & 1.3 & 1.9 & 2.1 & 2.3 & 1.8 & 2.7 & 2.1 \\
\hline $\mathrm{Fe}$ & 3 & 3.1 & 3.1 & 4.7 & 14 & 2.6 & 15 & 14 \\
\hline $\mathbf{L i}$ & 3.4 & 5 & 3.2 & 3.1 & 5 & 4.4 & 6 & 3.6 \\
\hline $\mathbf{M g}$ & 9.1 & 9.1 & 9.0 & 9.0 & 9.1 & 8.9 & 9.0 & 9.0 \\
\hline $\mathbf{M n}$ & .4 & $<1$ & .2 & .3 & $<1$ & .2 & 1.1 & 1.0 \\
\hline Mo & 1.6 & $<10$ & 1.8 & 1.6 & $<10$ & 1.8 & $<10$ & 1.8 \\
\hline $\mathrm{Na}$ & 19 & 20 & 18.8 & 18.8 & 20 & 19.0 & 19 & 19.7 \\
\hline $\mathbf{P b}$ & .7 & $<1$ & $<.3$ & $<.3$ & $<1$ & $<3$ & $<1$ & $<.3$ \\
\hline $\mathrm{SiO}_{2}$ & 4.9 & 5.0 & 4.9 & 4.9 & 5.0 & 4.8 & 4.9 & 4.8 \\
\hline $\mathbf{S r}$ & 156 & 157 & 150 & 151 & 157 & 155 & 156 & 152 \\
\hline V & 1.3 & $<6$ & 1.2 & 1.2 & $<6$ & 1.1 & $<6$ & 1.2 \\
\hline $\mathrm{Zn}$ & 1.8 & 3.3 & 1.8 & .9 & 3.8 & 3.3 & 6.0 & 2.1 \\
\hline
\end{tabular}


Table 2.-Summary of selected trace-metal concentrations in the Mississippi River below Belle Chasse, Louisiana, June 28, 1989

[Samples collected processed, and analyzed by U.S. Geological Survey National Stream Quality Accounting Network (NASQAN), National Research Program (NRP), and Branch of Analytical (BAS). All constituents reported in micrograms per liter, except $\mathrm{Ca}, \mathrm{Mg}, \mathrm{Na}$, and $\mathrm{SiO}_{2}$, which are reported in milligrams per liter]

\begin{tabular}{|c|c|c|c|c|c|c|c|c|}
\hline \multirow{3}{*}{ 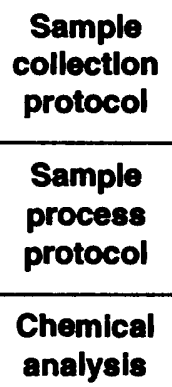 } & \multirow{3}{*}{$\begin{array}{l}\text { NRP } \\
\text { grab } \\
\text { NRP } \\
\text { NRP }\end{array}$} & \multicolumn{4}{|c|}{ NRP depth-integrated } & \multicolumn{3}{|c|}{ NASQAN depth-integrated } \\
\hline & & \multicolumn{2}{|c|}{ NRP } & \multirow{2}{*}{$\begin{array}{l}\text { NRP1 } \\
\text { NRP }\end{array}$} & \multirow{2}{*}{$\begin{array}{c}\text { NASRAN } \\
\text { BAS }\end{array}$} & \multirow{2}{*}{$\begin{array}{c}\text { NASQAN } \\
\text { NRP }\end{array}$} & \multirow{2}{*}{$\begin{array}{c}\text { NASQAN } \\
\text { BAS }\end{array}$} & \multirow{2}{*}{$\begin{array}{c}\text { NRP } \\
\text { NRP }\end{array}$} \\
\hline & & BAS & NRP & & & & & \\
\hline \multicolumn{9}{|l|}{ Constituent } \\
\hline $\mathbf{B a}$ & 52.8 & 50 & 52.9 & 53.3 & 48 & 51.4 & 50 & 48.2 \\
\hline $\mathrm{Be}$ & $<.02$ & $<.5$ & $<.02$ & $<.02$ & $<.5$ & $<.02$ & $<.5$ & .06 \\
\hline $\mathrm{Ca}$ & 32.6 & 33 & 32.5 & 32.7 & 32 & 32.6 & 33 & 32.7 \\
\hline Cd & .2 & $<.1$ & $<.1$ & $<.1$ & .2 & $<.2$ & .2 & $<.1$ \\
\hline Co & $<.03$ & $<1$ & $<.03$ & $<.03$ & $<1$ & .09 & $<1$ & .06 \\
\hline $\mathrm{Cr}$ & $<.2$ & $<5$ & $<.2$ & .2 & $<5$ & $<.2$ & $<5$ & $<.2$ \\
\hline $\mathrm{Cu}$ & 2 & 1.4 & 2.2 & 2.3 & 1.4 & 2.0 & 2.6 & 1.6 \\
\hline $\mathrm{Fe}$ & 2.6 & $<3$ & 2.3 & 1.7 & 12 & 8.5 & 11 & 4.1 \\
\hline $\mathrm{Li}$ & 3.2 & 6 & 3.5 & 3.3 & 5 & 3.2 & 5 & 3.2 \\
\hline $\mathbf{M g}$ & 9.3 & 9.5 & 9.3 & 9.4 & 9.2 & 9.3 & 9.4 & 9.3 \\
\hline $\mathbf{M n}$ & 1 & $<1$ & .2 & .4 & 1.7 & 1.1 & $<1$ & 1.1 \\
\hline Mo & 1.8 & $<10$ & 1.7 & 1.7 & $<10$ & 1.8 & $<10$ & 1.8 \\
\hline $\mathrm{Na}$ & 22.5 & 23 & 22.0 & 22.1 & 22 & 22.5 & 23 & 22.6 \\
\hline $\mathbf{P b}$ & $<3$ & $<1$ & $<0.3$ & $<3$ & $<1$ & $<.3$ & $<1$ & $<.3$ \\
\hline $\mathrm{SiO}_{2}$ & 5 & 5.1 & 5.0 & 5.0 & 5.0 & 5.0 & 5.1 & 4.9 \\
\hline $\mathbf{S r}$ & 158 & 160 & 158 & 151 & 156 & 161 & 161 & 160 \\
\hline V & 1.6 & $<6$ & 1.5 & 1.4 & $<6$ & 1.4 & $<6$ & 1.3 \\
\hline $\mathrm{Zn}$ & 2.3 & 3.5 & 2.0 & 2.0 & 5.5 & 2.3 & 3.7 & 1.2 \\
\hline
\end{tabular}




\section{DATA COLLECTION, SAMPLE PROCESSING, AND CHEMICAL-ANALYSIS PROTOCOLS}

Subsequent to the preliminary experiment discussed in the previous section, a more detailed comparison study, using NRP and NASQAN protocols, was designed to attempt to identify specific areas where problems, such as contamination, are prevalent. A sampling cruise of the ACADIANA in June 1990 was dedicated to collection of data for intensive comparative analysis of dissolved trace-metal sampling, processing, and analysis results between NRP and NASQAN personnel at several NASQAN sampling sites. The rivers sampled, the NASQAN site, and the NASQAN project office performing the sampling are listed in table 3. Samples were collected and split according to the scheme outlined in figure 3. In addition to the comparison between NRP and NASQAN project offices, the University of Southern Mississippi (USM) also collected samples at the same times and locations and analyzed subsample splits for selected trace metals to provide supplementary comparative data.

Table 3.-U.S. Geological Survey National Stream Quality Accounting Network sampling sites with corresponding District offices

\begin{tabular}{lll}
\hline \multicolumn{1}{c}{ River } & \multicolumn{1}{c}{ NASQAN site } & District \\
\hline Illinois & Valley City, Ill. & Illinois \\
Mississippi & Grafton, Ill. & Missouri \\
Mississippi & Thebes, Mo. & Missouri \\
Ohio & Lock and Dam 53, Ill. & Kentucky \\
Mississippi & Memphis, Tenn. & Arkansas \\
Mississippi & Arkansas City, Ark. & Arkansas \\
Yazoo & Redwood, Miss. & Mississippi \\
Mississippi & Vicksburg, Miss. & Mississippi \\
Mississippi & St. Francisville, La. & Louisiana \\
Mississippi & Belle Chasse, La. & Louisiana \\
\hline
\end{tabular}




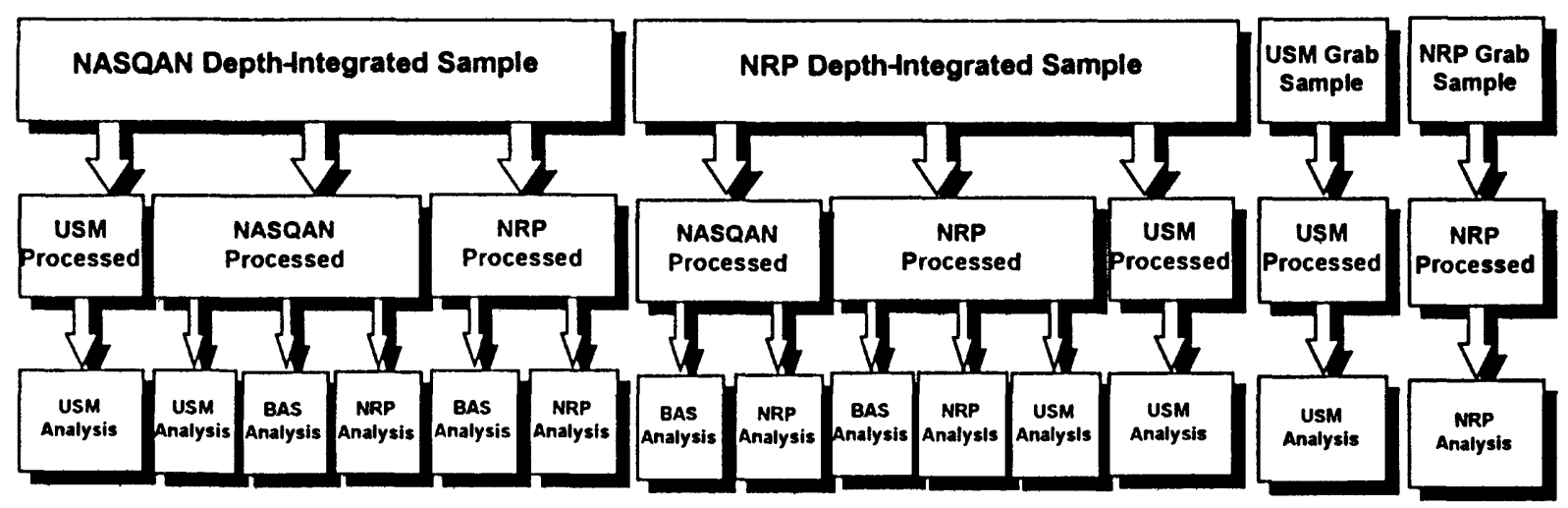

Figure 3. Sample collection, processing, and chemical-analysis protocols for June 1990 comparison study in the Mississippi River and some of its tributaries. NRP, NASQAN, and BAS represent U.S. Geological Survey National Research Program, National Stream Quality Accounting Network and Branch of Analytical Services, respectively. USM represents the University of Southern Mississippi.

The USM's sample-collection protocol consisted of collecting a grab sample (at the centroid of discharge of the river) with an acid-cleaned linear polyethylene bottle attached to the end of a long nonmetallic pole. The bottle was immersed upside-down below the river surface on the upstream side of the ship, inverted, and allowed to fill. Samples were filtered through a 0.4- $\mu \mathrm{m}$ Nuclepore filter after cleaning and flushing, using a clean filtration system described in (Boyle and others, 1982). Samples were acidified to $\mathrm{pH} 1.8$ with double quartz/Tefion-distilled HCl. Analytical procedures used at the University of Southern Mississippi consisted of electrothermal-vaporization atomic absorption spectrometry after preconcentrating the trace metals by using a cobaltpyrrolidine dithiocarbamate coprecipitation technique (Boyle and others, 1981; Shiller and Boyle, 1987).

\section{COMPARISON OF TRACE-METAL DATA}

The results of the NRP and NASQAN trace-metal comparative study at each sampling site are listed in tables 4-13 [see Moody and Meade (1993), for more details on location of the sampling sites], and the collection-affected and processing-affected concentrations for $\mathrm{Al}, \mathrm{Cd}, \mathrm{Cr}, \mathrm{Cu}, \mathrm{Pb}, \mathrm{Zn}$, and $\mathrm{Fe}$ are graphically shown in figures 4-10, respectively. Each figure shows concentrations of trace metals in samples collected through the NASQAN program and the NRP. Each plot includes a superimposed indication of reporting limits for each determination. In each figure the collectionaffected samples are depicted by the open-circle symbols; these represent samples that were collected by NASQAN project personnel and processed and analyzed by NRP personnel, plotted against similar samples collected, processed, and analyzed by NRP personnel. Similarly, in the same figures, the process-affected samples are depicted by triangles; these represent samples that were collected by NRP personnel, processed by NASQAN personnel, and subsequently analyzed by NRP personnel, plotted against samples collected, processed and analyzed by NRP personnel. 
Table 4.-Summary of selected trace-metal concentrations in the Illinois River at Valley City, illinois, June 7, 1990

[Samples collected, processed, and analyzed by U.S. Geological Survey National Stream Quality Accounting Network (NASQAN), National Research Program (NRP), Branch of

Analytical Services (BAS), and the University of Southern Mississippi (USM). All constituents reported in micrograms per liter, except $\mathrm{Ca}, \mathrm{Mg}, \mathrm{Na}$, and $\mathrm{SiO}_{2}$, which are reported in milligrams per liter. The -- indicate constituents not measured]

\begin{tabular}{|c|c|c|c|c|c|c|c|c|c|c|c|c|c|c|}
\hline \multirow{3}{*}{$\begin{array}{c}\text { Collec- } \\
\text { tion }\end{array}$} & \multicolumn{6}{|c|}{ NASQAN depth-Integrated } & \multicolumn{6}{|c|}{ NRP depth-Integrated } & \multirow{3}{*}{$\begin{array}{c}\begin{array}{l}\text { USM } \\
\text { grab }\end{array} \\
\text { USM } \\
\text { USM }\end{array}$} & \multirow{3}{*}{$\begin{array}{c}\begin{array}{l}\text { USM } \\
\text { grab }\end{array} \\
\text { NRP } \\
\text { NRP }\end{array}$} \\
\hline & \multirow{2}{*}{$\begin{array}{c}\text { USM } \\
\text { USM }\end{array}$} & \multicolumn{3}{|c|}{ NASQAN } & \multicolumn{2}{|c|}{ NRP } & \multicolumn{2}{|c|}{ NASQAN } & \multicolumn{2}{|c|}{ NRP } & \multirow{2}{*}{$\begin{array}{l}\text { NRP } \\
\text { USM }\end{array}$} & \multirow{2}{*}{$\begin{array}{c}\text { USM } \\
\text { USM }\end{array}$} & & \\
\hline & & USM & BAS & NRP & BAS & NRP & BAS & NRP & BAS & NRP & & & & \\
\hline \multicolumn{15}{|l|}{$\begin{array}{l}\text { Consti- } \\
\text { tuent }\end{array}$} \\
\hline $\mathrm{Ag}$ & -- & -- & $<1$ & .- & $<1$ & -- & 1.0 & -- & $<1$ & -- & -- & -- & -- & -- \\
\hline $\mathrm{Al}$ & 6.5 & 8.5 & -- & 23 & - & -- & -- & 33 & -- & 10 & 3.4 & 1.8 & 3.2 & 7.4 \\
\hline As & - & -- & - & 1.6 & -- & - & -- & 1.8 & - & .71 & - & - & - & 1.3 \\
\hline B & -- & - & -- & 140 & -- & -- & - & 150 & -- & 96 & -- & - & -- & 87 \\
\hline $\mathrm{Ba}$ & -- & - & 73 & 70 & 64 & -- & 76 & 72 & 65 & 64 & -- & -- & -- & 63 \\
\hline $\mathrm{Be}$ & -- & -- & $<.5$ & .09 & $<.5$ & -. & $<.5$ & .13 & $<.5$ & $<.02$ & -- & -- & -- & .03 \\
\hline $\mathrm{Ca}$ & -- & -- & 77 & 75 & 77 & - & 78 & 75 & 78 & -- & -. & -. & -- & - \\
\hline $\mathrm{Cd}$ & -- & -- & $<1$ & $<.1$ & $<1$ & - & $<1$ & .20 & $<1$ & $<.01$ & $>.2$ & .03 & .027 & $<.1$ \\
\hline Co & -- & - & $<3$ & .68 & $<3$ & - & $<3$ & .66 & $<3$ & .61 & -- & -- & -- & .54 \\
\hline $\mathrm{Cr}$ & .- & -. & 1.1 & 2.2 & $<.5$ & - & $<.5$ & 2.7 & $<.5$ & .18 & -- & -- & -- & .21 \\
\hline $\mathrm{Cu}$ & 1.8 & 1.4 & 2.5 & 2.7 & 1.7 & .. & 1.5 & 2.6 & 1.4 & 1.5 & 1.7 & 1.3 & 1.3 & 1.6 \\
\hline $\mathrm{Fe}$ & 1.5 & 4.5 & 6.9 & -- & 3.1 & -- & 14 & -- & 7.8 & -- & 1.0 & 1.2 & 1.5 & -. \\
\hline $\mathrm{Li}$ & -- & - & 9.6 & 5.4 & 9.9 & -- & 9.8 & 5.3 & 10 & 4.3 & -- & - & -- & 4.7 \\
\hline $\mathbf{M g}$ & - & - & 31 & 32 & 31 & - & 31 & 31 & 31 & -- & -- & 33 & 33 & -- \\
\hline $\mathbf{M n}$ & -. & - & 1.3 & 2.5 & $<1$ & -- & 1.4 & 3.1 & 1.2 & 3 & - & - & -- & 1.4 \\
\hline Mo & 3.4 & 2.8 & $<10$ & 3.8 & $<10$ & -- & $<10$ & 4.1 & $<10$ & 3.7 & 3.7 & 3.7 & 3.1 & 3.8 \\
\hline $\mathrm{Na}$ & - & - & 25 & 25 & 26 & -- & 25 & 25 & 25 & -. & -- & - & -. & -- \\
\hline $\mathrm{Ni}$ & -- & 1.7 & $<10$ & -- & $<10$ & - & $<10$ & -- & $<10$ & -- & 3.0 & 2.4 & 2.6 & -- \\
\hline $\mathbf{P b}$ & - & -- & $<.5$ & 1.1 & $<.5$ & -- & $<.5$ & .33 & $<.5$ & .08 & -- & -- & -- & $<.06$ \\
\hline Se & - & -- & -- & 2.5 & -- & -- & -- & 4 & - & 2.7 & -- & -- & -- & .43 \\
\hline $\mathrm{SiO}_{2}$ & -- & -- & 6.2 & 6.3 & 6.2 & -- & 6.2 & 6.5 & 6.2 & -- & 7.0 & 6.6 & 6.4 & -- \\
\hline $\mathrm{Sr}$ & -- & -- & 201 & 221 & 202 & -- & 202 & 221 & 202 & 217 & -. & -- & -. & 217 \\
\hline $\mathbf{T}$ & - & -. & -- & $<.05$ & -. & -- & -- & .07 & -- & $<.05$ & -- & -- & -- & $<.05$ \\
\hline $\mathbf{U}$ & -- & -- & -- & 2 & .- & -- & .- & 2 & -- & 1.8 & -- & -- & -- & 1.7 \\
\hline v & 1.7 & 1.4 & $<6$ & 2.2 & $<6$ & -- & $<6$ & 2.4 & $<6$ & 1.9 & 1.8 & 1.8 & 1.8 & 1.8 \\
\hline $\mathrm{Zn}$ & 1.1 & 5.2 & 6.1 & 3.5 & $<3$ & -. & 200 & 3.4 & $<3$ & 2.2 & 1.8 & 1.4 & 1.0 & 2.4 \\
\hline
\end{tabular}


Table 5.-Summary of selected trace-metal concentrations in the Mississippi River below Grafton, Illinois, June 11, 1990

[Samples collected, processed, and analyzed by U.S. Geological Survey National Stream Quality Accounting Network (NASQAN), National Research Program (NRP), Branch of Analytical Services (BAS), and the University of Southern Mississippi (USM). All constituents reported in micrograms per liter, except $\mathrm{Ca}, \mathrm{Mg}, \mathrm{Na}$, and $\mathrm{SiO}_{2}$, which are reported in milligrams per liter. The -- indicate constituents not measured]

\begin{tabular}{|c|c|c|c|c|c|c|c|c|c|c|c|c|c|c|}
\hline \multirow{3}{*}{$\begin{array}{c}\begin{array}{c}\text { Collec- } \\
\text { tlon }\end{array} \\
\begin{array}{l}\text { Pro- } \\
\text { cess }\end{array} \\
\begin{array}{l}\text { Anai- } \\
\text { ysis }\end{array}\end{array}$} & \multicolumn{6}{|c|}{ NASQAN depth-Integrated } & \multicolumn{6}{|c|}{ NRP depth-Integrated } & \multirow{3}{*}{$\begin{array}{c}\begin{array}{c}\text { USM } \\
\text { grab }\end{array} \\
\text { USM } \\
\text { USM }\end{array}$} & \multirow{3}{*}{$\begin{array}{l}\text { USM } \\
\text { grab } \\
\text { NRP } \\
\text { NRP }\end{array}$} \\
\hline & \multirow{2}{*}{ USM } & \multicolumn{3}{|c|}{ NASQAN } & \multicolumn{2}{|c|}{ NRP } & \multicolumn{2}{|c|}{ NASQAN } & \multicolumn{2}{|c|}{ NRP } & \multirow{2}{*}{$\begin{array}{l}\text { NRP } \\
\text { USM }\end{array}$} & \multirow{2}{*}{$\begin{array}{c}\text { USM } \\
\text { USM }\end{array}$} & & \\
\hline & & USM & BAS & NRP & BAS & NRP & BAS & NRP & BAS & NRP & & & & \\
\hline \multicolumn{15}{|l|}{$\begin{array}{c}\text { Consti- } \\
\text { tuent }\end{array}$} \\
\hline $\mathrm{Ag}$ & - & - & 1.0 & - & $<1$ & -- & $<1$ & -- & $<1$ & -- & -- & -- & -- & -- \\
\hline Al & 21 & -- & - & 19 & - & 9.1 & -- & 27 & -- & 5.8 & 7.6 & 4.9 & 4.1 & 17 \\
\hline As & - & - & - & 1.7 & - & 1.4 & -- & 1.3 & $-\cdot$ & 1 & -- & -- & -- & .78 \\
\hline B & - & - & -. & 53 & - & 42 & - & 60 & -. & 33 & -- & -. & -- & 37 \\
\hline $\mathrm{Ba}$ & - & - & 81 & 78 & 80 & 77 & 79 & 77 & 81 & 76 & - & - & -- & 78 \\
\hline $\mathrm{Be}$ & - & - & $<.5$ & $<.02$ & $<.5$ & $<.02$ & $<0.5$ & .04 & $<.5$ & $<.02$ & - & - & -- & .05 \\
\hline $\mathrm{Ca}$ & -- & - & 47 & 46 & 48 & 35 & 49 & 37 & 53 & 50 & -- & - & -- & -. \\
\hline $\mathrm{Cd}$ & .04 & - & 1.2 & .18 & $<1$ & $<.1$ & 1.2 & $<.1$ & $<1$ & $<.1$ & .16 & -- & .014 & $<.1$ \\
\hline Co & -- & - & $<3$ & .36 & $<3$ & .39 & $<3$ & .43 & $<3$ & .38 & -- & -- & -- & 38 \\
\hline $\mathrm{Cr}$ & - & - & .56 & .68 & $<50$ & .28 & $<.50$ & .42 & $>.50$ & $<.2$ & - & -. & -- & $<.2$ \\
\hline $\mathrm{Cu}$ & - & -- & 4.9 & 4 & 2.8 & 3.1 & 2.3 & 2.8 & 1.6 & 2.1 & 2.4 & 1.5 & 1.6 & 1.4 \\
\hline $\mathrm{Fe}$ & 3 & - & 24 & - & 6.5 & - & 18 & - & 21 & -. & 2.6 & 2.4 & 2.1 & - \\
\hline $\mathbf{L i}$ & - & -- & 6.6 & 3 & 7.1 & 3.3 & 5.9 & 3.7 & 5.7 & 3.7 & - & -- & -- & 3.5 \\
\hline $\mathbf{M g}$ & -- & - & 15 & 16 & 16 & 12 & 16 & 13 & 18 & 18 & - & 18 & 18 & -- \\
\hline $\mathrm{Mn}$ & - & - & 3.7 & 2 & $<1$ & .45 & 2.5 & 1.5 & $<1$ & .41 & - & -. & -- & 1 \\
\hline Mo & - & -. & $<10$ & 1 & $<10$ & 1.8 & $<10$ & 1.9 & $<10$ & 1.9 & 1.6 & -- & 1.6 & 1.8 \\
\hline $\mathrm{Na}$ & - & - & 9.9 & 9 & 9.9 & 7.6 & 11 & 8.4 & 12 & 11 & - & -- & -- & - \\
\hline $\mathrm{Ni}$ & 2.0 & - & $<10$ & - & $<10$ & - & $<10$ & - & $<10$ & -. & 2.1 & 1.8 & 1.9 & -- \\
\hline $\mathbf{P b}$ & - & -- & .56 & $<.60$ & $<50$ & $<.06$ & $<.50$ & $<.06$ & $<.50$ & $<.06$ & -- & -- & -- & $<.06$ \\
\hline $\mathrm{Se}$ & - & -- & -- & $<3$ & - & $<3$ & - & $<3$ & - & $<3$ & -- & - & -- & $<3$ \\
\hline $\mathrm{SiO}_{2}$ & -- & -- & 6.7 & 6 & 6.5 & 5.1 & 6.8 & 5.1 & 6.6 & 6.7 & -- & -- & -- & -- \\
\hline $\mathrm{Sr}$ & - & -- & 112 & 115 & 126 & 118 & 136 & 126 & 137 & 6.6 & 6.7 & 6.7 & 67 & 125 \\
\hline $\mathrm{Tl}$ & -- & - & - & $<.05$ & -- & $<.05$ & -- & $<.05$ & -- & $<.05$ & -. & - & -- & $<.05$ \\
\hline $\mathbf{U}$ & - & -- & - & 1.8 &.- & 1.9 & -- & 1.8 & -- & 1.8 & -. & -. & -- & 1.8 \\
\hline$v$ & 2.4 & - & $<6$ & 2 & $<6$ & 1.9 & $<6$ & 2 & $<6$ & 1.7 & 1.6 & 1.9 & 1.7 & 1.9 \\
\hline $\mathrm{Zn}$ & .38 & -- & 12 & 13 & 7.8 & 3.2 & 16 & 11 & $<3$ & .94 & .92 & .28 & .31 & 1 \\
\hline
\end{tabular}


Table 6.-Summary of seiected trace-metai concentrations In the Mississippi River at Thebes, ilinois, June 13, 1990

[Samples collected, processed, and analyzed by U.S. Geological Survey National Stream Quality Accounting Network (NASQAN), National Research Program (NRP), Branch of

Analytical Services (BAS), and the University of Southern Mississippi (USM). All constituents reported in micrograms per liter, except $\mathrm{Ca}, \mathrm{Mg}, \mathrm{Na}$, and $\mathrm{SiO}_{2}$, which are reported in milligrams per liter. The -- indicate constituents not measured]

\begin{tabular}{|c|c|c|c|c|c|c|c|c|c|c|c|c|c|c|}
\hline \multirow{3}{*}{$\begin{array}{c}\begin{array}{c}\text { Collec- } \\
\text { tion }\end{array} \\
\text { Pro- } \\
\text { cess } \\
\begin{array}{c}\text { Anal- } \\
\text { ysis }\end{array}\end{array}$} & \multicolumn{6}{|c|}{ NASQAN depth-integrated } & \multicolumn{6}{|c|}{ NRP depth-integrated } & \multirow{3}{*}{$\begin{array}{c}\begin{array}{l}\text { USM } \\
\text { grab }\end{array} \\
\text { USM } \\
\text { USM }\end{array}$} & \multirow{3}{*}{$\begin{array}{c}\begin{array}{l}\text { USM } \\
\text { grab }\end{array} \\
\text { NRP } \\
\text { NRP }\end{array}$} \\
\hline & \multirow{2}{*}{$\begin{array}{c}\text { USM } \\
\text { USM }\end{array}$} & \multicolumn{3}{|c|}{ NASQAN } & \multicolumn{2}{|c|}{ NRP } & \multicolumn{2}{|c|}{ NASQAN } & \multicolumn{2}{|c|}{ NRP } & \multirow{2}{*}{$\begin{array}{l}\text { NRP } \\
\text { USM }\end{array}$} & \multirow{2}{*}{$\begin{array}{c}\text { USM } \\
\text { USM }\end{array}$} & & \\
\hline & & USM & BAS & NRP & BAS & NRP & BAS & NRP & BAS & NRP & & & & \\
\hline \multicolumn{15}{|l|}{$\begin{array}{c}\text { Consti- } \\
\text { tuent }\end{array}$} \\
\hline $\mathbf{A g}$ & -- & -- & $<1$ & -- & $<1$ & -- & $<1$ & - & $<1$ & -- & -- & -- & - & -- \\
\hline $\mathrm{Al}$ & -- & 74 & - & 53 & -- & 13 & -- & 20 & -- & 11 & 4.9 & -- & 2.3 & 6.5 \\
\hline As & - & - & - & 1.7 & - & 2 & - & 1.9 & -- & 1.7 & -- & -- & -- & 1.4 \\
\hline B & - & -- & - & 45 & - & 47 & -. & 50 & -- & 36 & -- & -- & -. & 36 \\
\hline $\mathrm{Ba}$ & - & - & 81 & 71 & 80 & 79 & 72 & 77 & 78 & 78 & -- & - & -- & 83 \\
\hline $\mathrm{Be}$ & -- & - & $<.5$ & $<.02$ & $<.5$ & $<.02$ & $<.5$ & .02 & $<.5$ & .03 & -- & -- & -- & .10 \\
\hline $\mathrm{Ca}$ & - & -- & 40 & 35 & 40 & 39 & 35 & 39 & 40 & 39 & -- & -- & -- & -- \\
\hline $\mathrm{Cd}$ & - & -- & $<1$ & $<.1$ & $<1$ & $<1$ & $<1$ & $<.1$ & 1.1 & $<.1$ & .13 & .022 & .018 & $<.1$ \\
\hline Co & - & - & $<3$ & .28 & $<3$ & .30 & $<3$ & .32 & $<3$ & .32 & -- & .. & -. & .28 \\
\hline $\mathrm{Cr}$ & -- & -- & $<.5$ & .37 & $<.5$ & .42 & $<.5$ & .30 & $<.5$ & $<.2$ & -- & -. & -. & 1.4 \\
\hline $\mathrm{Cu}$ & 3.2 & 5.0 & 4.6 & 5.7 & 2.9 & 3.7 & 4.1 & 3.4 & 1.6 & 2.2 & 2.1 & 1.9 & 1.7 & 1.6 \\
\hline $\mathrm{Fe}$ & 3.0 & $>30$ & 9.2 & - & 4.7 & -- & 22 & -- & 5.4 & - & 2.7 & 3.2 & 3.1 & -- \\
\hline $\mathbf{L i}$ & -- & - & 7.7 & 5.1 & 7.7 & 5.7 & 6.6 & 5.6 & 7.9 & 5.1 & - & - & -. & 4.9 \\
\hline $\mathbf{M g}$ & -- & - & 12 & 1.5 & 12 & 12 & 10 & 12 & 12 & 12 & -- & 12 & 12 & -- \\
\hline $\mathbf{M n}$ & - & - & 1.2 & 2.4 & $<1$ & .34 & 1.9 & 1.3 & $<1$ & .36 & -- & -- & - & .52 \\
\hline Mo & 1.6 & 1.5 & $<10$ & 1.7 & $<10$ & 1.8 & $<10$ & 1.8 & $<10$ & 1.7 & 1.6 & 1.8 & 1.5 & 1.9 \\
\hline $\mathrm{Na}$ & - & - & 12 & 11.4 & 12 & 13 & 11 & 13 & 12 & 13 & -- & -- & -- & -- \\
\hline $\mathrm{Ni}$ & 1.8 & 1.7 & $<10$ & -- & $<10$ & -- & $<10$ & -- & $<10$ & -- & 1.9 & 2.0 & 1.9 & -- \\
\hline $\mathrm{Pb}$ & -- & - & $<.5$ & .19 & $<.5$ & .08 & $<.5$ & .16 & $<.5$ & $<.06$ & -- & -- & -- & $<.06$ \\
\hline $\mathrm{Se}$ & -- & -- & - & $<3$ & -- & $<3$ & -- & $<3$ & -- & $<3$ & -. & -- & -- & $<3$ \\
\hline $\mathrm{SiO}_{2}$ & -- & - & 7.1 & 7.2 & 7.1 & 7.5 & 6.7 & 7.4 & 7.1 & 7.4 & 7.6 & 7.6 & 7.7 & -- \\
\hline $\mathrm{Sr}$ & - & - & 151 & 155 & 151 & 169 & 132 & 167 & 151 & 156 & -- & -- & -- & 155 \\
\hline $\mathrm{Tl}$ & -- & - & - & $<.05$ & -- & $<.05$ & -- & $<.05$ & -- & $<.05$ & -- & -- & -- & $<.05$ \\
\hline $\mathbf{U}$ & - & - & - & 1.3 & - & 1.5 & - & 1.5 & -- & 1.5 & - & - & -- & 1.4 \\
\hline $\mathbf{v}$ & 2.1 & 1.7 & $<6$ & 2.2 & $<6$ & 2.4 & $<6$ & 2.4 & $<6$ & 2.4 & 2.1 & 2.2 & 1.9 & 1.9 \\
\hline $\mathrm{Zn}$ & .26 & $>10$ & 6.9 & 9.8 & $<3$ & 7.5 & 4.2 & 7.5 & $<3$ & .88 & .22 & .18 & .19 & .70 \\
\hline
\end{tabular}


Table 7.-Summary of selected trace-metal concentrations in the Ohio River at Olmsted, Illinois, June 14, 1990

[Samples collected, processed, and analyzed by U.S. Geological Survey National Stream Quality Accounting Network (NASQAN), National Research Program (NRP), Branch of

Analytical Services (BAS), and the University of Southern Mississippi (USM). All constituents reported in micrograms per liter, except $\mathrm{Ca}, \mathrm{Mg}, \mathrm{Na}$, and $\mathrm{SiO}_{2}$, which are reported in milligrams per liter. The -- indicate constituents not measured]

\begin{tabular}{|c|c|c|c|c|c|c|c|c|c|c|c|c|c|c|}
\hline \multirow{3}{*}{$\begin{array}{c}\text { Collec- } \\
\text { tion }\end{array}$} & \multicolumn{6}{|c|}{ NASQAN depth-integrated } & \multicolumn{6}{|c|}{ NRP depth-integrated } & \multirow{3}{*}{$\begin{array}{c}\text { USM } \\
\text { grab } \\
\text { USM } \\
\text { USM }\end{array}$} & \multirow{3}{*}{$\begin{array}{c}\begin{array}{l}\text { USM } \\
\text { grab }\end{array} \\
\text { NRP } \\
\text { NRP }\end{array}$} \\
\hline & \multirow{2}{*}{$\begin{array}{c}\text { USM } \\
\text { USM }\end{array}$} & \multicolumn{3}{|c|}{ NASQAN } & \multicolumn{2}{|c|}{ NRP } & \multicolumn{2}{|c|}{ NASQAN } & \multicolumn{2}{|c|}{ NRP } & \multirow{2}{*}{$\begin{array}{c}\text { NRP } \\
\text { USM }\end{array}$} & \multirow{2}{*}{$\begin{array}{c}\text { USM } \\
\text { USM }\end{array}$} & & \\
\hline & & USM & BAS & NRP & BAS & NRP & BAS & NRP & BAS & NRP & & & & \\
\hline \multicolumn{15}{|l|}{$\begin{array}{l}\text { Consti- } \\
\text { tuent }\end{array}$} \\
\hline $\mathrm{Ag}$ & - & $-\cdots$ & $<1$ & - & - & - & $<1$ & - & $<1$ & - & -- & -. & -. & - \\
\hline Al & 5.6 & 91 & -- & 45 & - & 13 & -- & 56 & -- & 11 & -- & - & 6.7 & 7.6 \\
\hline As & -- & -- & - & .90 & - & .92 & -- & .95 & - & 1.1 & -- & -- & -- & .92 \\
\hline B & -- & -. & -- & 50 & -- & 35 & -. & 55 & -. & 30 & -- & - & -- & 37 \\
\hline $\mathrm{Ba}$ & .- & - & 41 & 39 & -- & 32 & 42 & 37 & 31 & 30 & -- & -- & -- & 32 \\
\hline $\mathrm{Be}$ & -- & .- & $<.5$ & $<.02$ & - & $<.02$ & $<.5$ & .04 & $<.5$ & .02 & -- & .- & .- & $<.02$ \\
\hline $\mathrm{Ca}$ & -. & -- & 34 & 34 & -- & 34 & 34 & 34 & 34 & 35 & -. & -. & -. & .. \\
\hline $\mathrm{Cd}$ & .072 & .2 & $<1$ & .19 & -. & $<.1$ & $<1$ & 1.3 & $<1$ & $<.1$ & $>.2$ & -- & .010 & $<.1$ \\
\hline Co & - & -- & $<3$ & .33 &.- & .34 & $<3$ & .32 & $<3$ & .31 & -. & - & -- & .26 \\
\hline $\mathrm{Cr}$ & -- & -- & 1.6 & 1.9 & -- & $<.2$ & 2.2 & 2.3 & 2.1 & $<.2$ & -- & - & -. & $<.2$ \\
\hline $\mathrm{Cu}$ & -. & $>9$ & $<10$ & 8.1 & -- & 6.9 & $<10$ & 2 & $<10$ & 1.5 & 1.7 & -- & 1.4 & 1.4 \\
\hline $\mathrm{Fe}$ & 2.2 & $>28$ & 28 & -- & -- & -. & 42 & -- & 4.7 & -- & 2.1 & -- & 2.1 & -- \\
\hline $\mathrm{Li}$ & -- & -- & 5.9 & 1.9 & -- & 1.9 & 4.8 & 2 & 5.1 & 1.8 & - & - & -- & 2.2 \\
\hline $\mathbf{M g}$ & -- & -- & 7.8 & 8.1 & - & 8.1 & 7.7 & 8.1 & 7.9 & 8.4 & - & 8.3 & 8.6 & -- \\
\hline $\mathbf{M n}$ & .. & - & 3.9 & 5.4 & -- & 1.8 & 2.6 & 1.7 & $<1$ & .19 & -- & -- & -- & .64 \\
\hline Mo & 1.2 & 1.2 & $<10$ & 1.4 & -- & 1.4 & $<10$ & 1.4 & $<10$ & 1.5 & 1.4 & - & 1.3 & 1.5 \\
\hline $\mathrm{Na}$ & -- & -- & 6.8 & 7 & - & 7.1 & 6.8 & 7.1 & 6.8 & 7.3 & -- & -. & -- & -- \\
\hline $\mathrm{Ni}$ & 1.9 & 1.6 & $<10$ & -- & - & -- & $<10$ & -- & $<10$ & -- & -- & -- & 1.2 & -- \\
\hline $\mathbf{P b}$ & -- & -- & 16 & 8 & - & 3.3 & 2.7 & 1.3 & $<.5$ & $<.06$ & -- & -- & -- & $<.06$ \\
\hline $\mathrm{Se}$ & -- & - & -- & $<3$ & -- & $<3$ & -- & $<3$ & -- & $<3$ & -- & -- & -- & $<3$ \\
\hline $\mathrm{SiO}_{2}$ & - & - & 5.3 & 5.7 & -- & 5.7 & 5.3 & 5.7 & 5.3 & 6 & 5.6 & 5.6 & 5.8 & -. \\
\hline $\mathrm{Sr}$ & - & -. & 118 & 140 & -- & 139 & 118 & 137 & 118 & 140 & -- & -- & -- & 134 \\
\hline $\mathrm{Tl}$ & -- & -- & -- & $<.05$ & -- & $<.05$ & -- & $<.05$ & -- & $<.05$ & - & -- & -- & $<.05$ \\
\hline $\mathbf{U}$ & -- & -- & - & .35 & -- & .38 & -- & .37 & -- & .24 & -- & -. & -- & .29 \\
\hline $\mathrm{v}$ & .42 & .46 & $<6$ & .57 & - & .57 & $<6$ & .68 & $<6$ & .63 & .61 & - & .58 & .55 \\
\hline $\mathrm{Zn}$ & 1.2 & $>9$ & 5.3 & 49 &.- & 3.8 & 4.4 & 44 & $<3$ & .87 & .39 & -- & .19 & .83 \\
\hline
\end{tabular}


Tabie 8.-Summary of seiected trace-metal concentrations in the Mississippi River beiow Memphis, Tennessee, June 18, 1990

[Samples collected, processed, and analyzed by U.S. Geological Survey National Stream Quality Accounting Network (NASQAN), National Research Program (NRP), Branch of Analytical Services (BAS), and the University of Southern Mississippi (USM). All constituents reported in micrograms per liter, except $\mathrm{Ca}, \mathrm{Mg}, \mathrm{Na}$, and $\mathrm{SiO}_{2}$, which are reported in milligrams per liter. The -- indicate constituents not measured]

\begin{tabular}{|c|c|c|c|c|c|c|c|c|c|c|c|c|c|c|}
\hline \multirow{3}{*}{$\begin{array}{c}\begin{array}{c}\text { Collec- } \\
\text { tion }\end{array} \\
\begin{array}{c}\text { Pro- } \\
\text { cess }\end{array} \\
\begin{array}{c}\text { Anal- } \\
\text { ysis }\end{array}\end{array}$} & \multicolumn{6}{|c|}{ NASQAN depth-integrated } & \multicolumn{6}{|c|}{ NRP depth-integrated } & \multirow{3}{*}{$\begin{array}{c}\begin{array}{c}\text { USM } \\
\text { grab }\end{array} \\
\text { USM } \\
\text { USM }\end{array}$} & \multirow{3}{*}{$\begin{array}{c}\begin{array}{l}\text { USM } \\
\text { grab }\end{array} \\
\text { NRP } \\
\text { NRP }\end{array}$} \\
\hline & \multirow{2}{*}{$\begin{array}{c}\text { USM } \\
\text { USM }\end{array}$} & \multicolumn{3}{|c|}{ NASQAN } & \multicolumn{2}{|c|}{ NRP } & \multicolumn{2}{|c|}{ NASQAN } & \multicolumn{2}{|c|}{ NRP } & \multirow{2}{*}{$\begin{array}{c}\text { NRP } \\
\text { USM }\end{array}$} & \multirow{2}{*}{$\frac{\text { USM }}{\text { USM }}$} & & \\
\hline & & USM & BAS & NRP & BAS & NRP & BAS & NRP & BAS & NRP & & & & \\
\hline \multicolumn{15}{|l|}{$\begin{array}{c}\text { Consti- } \\
\text { tuent }\end{array}$} \\
\hline $\mathbf{A g}$ &.- & - & $<1$ & - & $<1$ & -- & $<1$ & -- & $<1$ & -- & -- & -- & -- & -- \\
\hline $\mathrm{Al}$ & -- & 19 & - & 23 & -- & 13 & - & 35 & -- & 11 & 7.6 & -- & 4.1 & 13 \\
\hline As & - & -- & - & 1.7 & -- & 1.4 & -- & 1.7 & -- & .94 & -- & -- & -- & 1.2 \\
\hline B & -- & -- & -- & 43 & -- & 39 & -- & 41 & -- & 39 & -. & -- & .- & 38 \\
\hline $\mathrm{Ba}$ & -- & - & 60 & 59 & 63 & 61 & 58 & 56 & 60 & 59 & -- & -- & -- & 62 \\
\hline $\mathrm{Be}$ & -- & -. & $<.5$ & .02 & $<.5$ & .04 & $<.5$ & .04 & $<.5$ & $<.02$ & .- & -- & -- & $<.02$ \\
\hline $\mathrm{Ca}$ & -- & -. & 37 & 37 & 39 & 29 & 37 & 36 & 38 & 38 & -- & -- & .. & -- \\
\hline $\mathrm{Cd}$ & -- & $<.2$ & 3.9 & 2.9 & 3.6 & 2.7 & 2.5 & .19 & $<1$ & $<.1$ & $>.2$ & -- & -- & $<.1$ \\
\hline Co & -- & -- & $<3$ & .18 & $<3$ & .20 & $<3$ & .16 & $<3$ & .19 & .- & -- & -- & .31 \\
\hline $\mathrm{Cr}$ & -- & - & 1.9 & 1.4 & $<.5$ & .20 & 2.0 & 2.5 & $<.5$ & $<.2$ & - & -. & -. & $<2$ \\
\hline $\mathrm{Cu}$ & - & $<10$ & 13 & 13 & 12 & 13 & 66 & 2.8 & 1.8 & 1.6 & 2.7 & 1.6 & -. & 1.6 \\
\hline $\mathrm{Fe}$ & .- & 20 & 22 & - & 6.9 & -- & 33 & -- & $<3$ & -- & 2.8 & 2.0 & -- & -- \\
\hline $\mathrm{Li}$ & -- & -- & 6.6 & 3.6 & 6.0 & 3.7 & 6.3 & 3.5 & 6.0 & 3.7 & -- & -- & -- & 3.5 \\
\hline $\mathbf{M g}$ & - & - & 9.8 & 10 & 9.9 & 8.0 & 9.9 & 10 & 10 & 11 & -- & 11.1 & 11.1 & -- \\
\hline $\mathbf{M n}$ & - & - & 1.7 & 1.9 & 1.8 & 1.2 & 1.8 & 1.8 & $<1$ & .30 & .. & -- & -- & .57 \\
\hline Mo & -- & .- & $<10$ & 1.6 & $<10$ & 1.8 & $<10$ & 1.7 & $<10$ & 1.7 & 1.7 & -- & -- & 1.8 \\
\hline $\mathrm{Na}$ & - & -- & 10 & 11 & 11 & 8.3 & 10 & 10 & 11 & 11 & -- & -- & - & - \\
\hline $\mathrm{Ni}$ & - & 1.3 & $<10$ & - & $<10$ & - & $<10$ & - & $<10$ & -- & 1.7 & 1.4 & - & -- \\
\hline $\mathrm{Pb}$ & - & - & $<5$ & .35 & $<50$ & .08 & .96 & .57 & $<.5$ & $<.06$ & -- & -- & -- & $<.06$ \\
\hline $\mathrm{Se}$ & -. & - & -- & $<3$ & -- & $<3$ & - & $<3$ & -- & $<3$ & - & - & -- & $<3$ \\
\hline $\mathrm{SiO}_{2}$ & - & - & 6.5 & 6.7 & 6.5 & 5.2 & 6.6 & 6.9 & 6.5 & 6.9 & 6.7 & 6.9 & -- & -. \\
\hline Sr & -- & -- & 139 & 152 & 148 & 159 & 140 & 151 & 146 & 161 & -. & -- & -- & 154 \\
\hline $\mathrm{Tl}$ & -- & -- & -- & $<.05$ & -- & $<.05$ & -- & $<.05$ & -- & $<.05$ & -- & -- & -- & .04 \\
\hline $\mathbf{U}$ & .- & - & .- & .93 & - & .97 & -- & 1.0 & - & .83 & -. & -. & .- & .91 \\
\hline $\mathrm{v}$ & -- & - & $<6$ & 1.5 & $<6$ & 1.6 & $<6$ & 1.5 & $<6$ & 1.5 & 1.5 & 1.9 & -- & 1.5 \\
\hline $\mathrm{Zn}$ & -. & $>10$ & 3.0 & 12 & $<3$ & 1.1 & 14 & 17 & $<3$ & .57 & 1.4 & .22 & -. & .76 \\
\hline
\end{tabular}


Table 9.-Summary of selected trace-metal concentrations in the Mississippi River below Arkansas City, Arkansas, June 20, 1990

[Samples collected, processed, and analyzed by U.S. Geological Survey National Stream Quality Accounting Network (NASQAN), National Research Program (NRP), Branch of Analytical Services (BAS), and the University of Southern Mississippi (USM). All constituents reported in micrograms per liter, except $\mathrm{Ca}, \mathrm{Mg}, \mathrm{Na}$, and $\mathrm{SiO}_{2}$, which are reported in milligrams per liter. The -- indicate constituents not measured]

\begin{tabular}{|c|c|c|c|c|c|c|c|c|c|c|c|c|c|c|}
\hline \multirow{3}{*}{$\begin{array}{c}\begin{array}{c}\text { Collec- } \\
\text { tion }\end{array} \\
\begin{array}{l}\text { Pro- } \\
\text { cess }\end{array} \\
\begin{array}{l}\text { Anal- } \\
\text { ysis }\end{array}\end{array}$} & \multicolumn{6}{|c|}{ NASQAN depth-Integrated } & \multicolumn{6}{|c|}{ NRP depth-integrated } & \multirow{3}{*}{$\begin{array}{c}\begin{array}{l}\text { USM } \\
\text { grab }\end{array} \\
\text { USM } \\
\text { USM }\end{array}$} & \multirow{3}{*}{$\begin{array}{c}\text { USM } \\
\text { grab } \\
\text { NRP } \\
\text { NRP }\end{array}$} \\
\hline & \multirow{2}{*}{$\frac{\text { USM }}{\text { USM }}$} & \multicolumn{3}{|c|}{ NASQAN } & \multicolumn{2}{|c|}{ NRP } & \multicolumn{2}{|c|}{ NASQAN } & \multicolumn{2}{|c|}{ NRP } & \multirow{2}{*}{$\begin{array}{c}\text { NRP } \\
\text { USM }\end{array}$} & \multirow{2}{*}{$\begin{array}{c}\text { USM } \\
\text { USM }\end{array}$} & & \\
\hline & & USM & BAS & NRP & BAS & NRP & BAS & NRP & BAS & NRP & & & & \\
\hline \multicolumn{15}{|l|}{$\begin{array}{c}\text { Consti- } \\
\text { tuent }\end{array}$} \\
\hline Ag & -- & - & $<1$ & - & $<1$ & - & 1.4 & -- & $<1$ & - & - & -- & - & -- \\
\hline Al & 18 & 60 & -- & 39 & -- & 10 & - & 41 & -- & 9.2 & 6 & 5.4 & 8.2 & 3.7 \\
\hline As & -. & - & -- & 1.6 & - & 1.7 & - & 1.8 & -- & 1 & -- & -- & - & 2.1 \\
\hline B & - & - & -- & 37 & - & 33 & - & 37 & - & 35 & - & -- & -- & 34 \\
\hline $\mathrm{Ba}$ & - & - & 55 & 54 & 58 & 56 & 53 & 52 & 56 & 55 & -- & -- & - & 60 \\
\hline $\mathrm{Be}$ & - & - & $<.5$ & $<.02$ & $<.5$ & $<.02$ & .5 & .02 & $<.5$ & $<.02$ & -- & -- & -- & $<02$ \\
\hline $\mathrm{Ca}$ & - & - & 35 & 35 & 37 & 37 & 34 & 34 & 37 & 37 & - & - & - & -- \\
\hline $\mathrm{Cd}$ & $>.2$ & .2 & 3.3 & 3 & 3.5 & 2.4 & 1.7 & .19 & $<1$ & $<.1$ & .18 & .017 & .013 & $<.1$ \\
\hline Co & -- & -- & $<3$ & $<.1$ & $<3$ & .15 & $<3$ & .16 & $<3$ & .17 & - & - & -- & .31 \\
\hline $\mathrm{Cr}$ & - & - & 2.2 & 2.4 & 1.6 & .22 & 2 & 2.2 & $<50$ & .22 & - & -. & - & .41 \\
\hline $\mathrm{Cu}$ & 4.3 & 5 & 5.6 & 7.1 & 5.1 & 4.9 & 2.4 & 2.3 & 1.7 & 1.8 & 2.1 & 1.6 & 1.6 & 1.6 \\
\hline $\mathrm{Fe}$ & 7.5 & $>30$ & 30 & - & 4.2 & .. & 35 & - & 5.9 & - & 1.7 & 1.7 & 1.7 & \\
\hline $\mathrm{Li}$ & - & - & 5.1 & 3.2 & 5.5 & 3.1 & 4.9 & 2.9 & 5.5 & 3.2 & -- & 10 & - & 3.3 \\
\hline $\mathbf{M g}$ & - & - & 9.3 & 10 & 9.9 & 10 & 9.1 & 9.3 & 9.8 & 10 & -- & 10 & 10.6 & 10.6 \\
\hline $\mathbf{M n}$ & - & - & 1.6 & 1.5 & 1.5 & 1.6 & 1.7 & 1.8 & $<1$ & .91 & - & - & - & 1 \\
\hline Mo & 1.4 & 1.2 & $<10$ & 1.5 & $<10$ & 1.6 & $<10$ & 1.5 & $<10$ & 1 & 1.3 & 1.5 & 1.3 & 1.7 \\
\hline $\mathrm{Na}$ & -- & -- & 9.9 & 10 & 10 & 11 & 9.7 & 10 & 10 & 11 & - & -- & - & -- \\
\hline $\mathrm{Ni}$ & 1.6 & 1.4 & $<10$ & - & $<10$ & - & $<10$ & -- & $<10$ & - & 1.4 & 1.3 & 1.4 & - \\
\hline $\mathbf{P b}$ & - & -- & $<.5$ & .68 & $<.5$ & $<.06$ & $<.5$ & .26 & $<.5$ & $<.06$ & - & - & - & $<.06$ \\
\hline $\mathrm{Se}$ & - & -. & - & $<3$ & - & $<3$ & - & $<3$ & - & $<3$ & - & - & - & $<3$ \\
\hline $\mathrm{SiO}_{2}$ & - & - & 6.6 & 6.9 & 6.5 & 6.9 & 6.6 & 6.9 & 6.5 & 6.9 & 7.0 & 6.6 & 6.4 & - \\
\hline $\mathbf{S r}$ & - & - & 133 & 146 & 139 & 150 & 130 & 143 & 139 & 147 & - & - & -- & 151 \\
\hline $\mathrm{Tl}$ & - & - & -. & $<.05$ & -. & $<.05$ & - & $<.05$ & -- & $<.05$ & - & - & -- & $<.05$ \\
\hline $\mathbf{U}$ & - & - & - & .8 & - & .87 & - & .81 & - & .81 & - & -- & -- & .64 \\
\hline $\mathbf{v}$ & 1.6 & 1.1 & $<6$ & 1.5 & $<6$ & 1.4 & $<6$ & 1.4 & $<6$ & 1.5 & 1.6 & 1.6 & 1.4 & 1.6 \\
\hline $\mathrm{Zn}$ & .41 & $>10$ & $<3$ & 10 & $<3$ & .79 & 9.8 & 16 & $<3$ & .91 & .29 & .19 & .19 & 70 \\
\hline
\end{tabular}


Table 10.-Summary of selected trace-metai concentrations in the Yazoo River beiow Steele Bayou, Mississippi, June 22, 1990

[Samples collected, processed, and analyzed by U.S. Geological Survey National Stream Quality Accounting Network (NASQAN), National Research Program (NRP), Branch of Analytical Services (BAS), and the University of Southern Mississippi (USM). All constituents reported in micrograms per liter, except $\mathrm{Ca}, \mathrm{Mg}, \mathrm{Na}$, and $\mathrm{SiO}_{2}$, which are reported in milligrams per liter. The -- indicate constituents not measured]

\begin{tabular}{|c|c|c|c|c|c|c|c|c|c|c|c|c|c|c|}
\hline \multirow{3}{*}{$\begin{array}{c}\begin{array}{c}\text { Coilec- } \\
\text { tion }\end{array} \\
\begin{array}{l}\text { Pro- } \\
\text { cess }\end{array} \\
\begin{array}{l}\text { Anai- } \\
\text { ysis }\end{array}\end{array}$} & \multicolumn{6}{|c|}{ NASQAN depth-integrated } & \multicolumn{6}{|c|}{ NRP depth-integrated } & \multirow{3}{*}{$\begin{array}{c}\begin{array}{l}\text { USM } \\
\text { grab }\end{array} \\
\text { USM } \\
\text { USM }\end{array}$} & \multirow{3}{*}{$\begin{array}{c}\text { USM } \\
\text { grab } \\
\text { NRP } \\
\text { NRP }\end{array}$} \\
\hline & \multirow{2}{*}{$\begin{array}{c}\text { USM } \\
\text { USM }\end{array}$} & \multicolumn{3}{|c|}{ NASQAN } & \multicolumn{2}{|c|}{ NRP } & \multicolumn{2}{|c|}{ NASQAN } & \multicolumn{2}{|c|}{ NRP } & \multirow{2}{*}{$\begin{array}{c}\text { NRP } \\
\text { USM }\end{array}$} & \multirow{2}{*}{$\begin{array}{c}\text { USM } \\
\text { USM }\end{array}$} & & \\
\hline & & USM & BAS & NRP & BAS & NRP & BAS & NRP & BAS & NRP & & & & \\
\hline \multicolumn{15}{|l|}{$\begin{array}{l}\text { Consti- } \\
\text { tuent }\end{array}$} \\
\hline $\mathrm{Ag}$ & - & -- & $<1$ & - & $<1$ & -- & $<1$ & -- & 1.2 & -- & -. & -- & -- & -- \\
\hline $\mathrm{Al}$ & - & 680 & -- & 200 & - & 9.1 & - & 290 & -- & 3.5 & -- & -- & 3.3 & 2.3 \\
\hline As & -- & - & -- & 5.9 & - & 4.3 & -- & 5.6 & - & 4.4 & -- & -- & -- & 5.1 \\
\hline B & - & -- & -- & 51 & - & 22 & -- & 55 & - & 22 & - & -- & -- & 24 \\
\hline $\mathrm{Ba}$ & - & -- & 96 & 73 & 35 & 34 & 57 & 57 & 34 & 33 & $-\cdot$ & -- & -- & 37 \\
\hline $\mathrm{Be}$ &.- & -- & $<.5$ & .04 & $<.5$ & .02 & $<.5$ & .05 & $<.5$ & $<.02$ & -- & -. & .. & $<.02$ \\
\hline $\mathrm{Ca}$ & -. & -- & 11 & 11 & 11 & 11 & 12 & 11 & 11 & 11 & -. & -. & -. & -. \\
\hline $\mathrm{Cd}$ & .041 & $>.2$ & 13 & 7.2 & $<1$ & $<.1$ & $<1$ & 1.2 & 1.1 & $<.1$ & $>.2$ & .013 & .008 & $<.1$ \\
\hline Co & - & -- & $<3$ & .20 & $<3$ & $<.02$ & $<3$ & .23 & $<3$ & .03 & -- & -- & -- & .15 \\
\hline $\mathrm{Cr}$ & .. & - & $<.5$ & .60 & $<.5$ & $<.2$ & $<.5$ & .81 & $<.5$ & $<.2$ & -- & -. & -. & $<.2$ \\
\hline $\mathrm{Cu}$ & 2.8 & 3.0 & 4 & 3.6 & $<.5$ & 2.9 & .64 & 2.2 & $<.5$ & 1.8 & 2.8 & 1.5 & 1.7 & 1.6 \\
\hline $\mathrm{Fe}$ & 6.7 & $>28$ & 240 & -- & 10 & -- & 230 & -. & 12 & -- & 6.8 & 8.9 & 8.1 & -- \\
\hline $\mathrm{Li}$ & -- & - & $<4$ & 1.6 & $<4$ & 1.5 & $<4$ & 1.6 & $<4$ & 1.5 & - & -. & -- & 1.6 \\
\hline $\mathbf{M g}$ & -- & - & 3.1 & 3.3 & 3.3 & 3.4 & 3.4 & 3.3 & 3.3 & 3.3 & - & 3.4 & 3.4 & -- \\
\hline $\mathbf{M n}$ & -- & -- & 84 & 77 & 62 & 58 & 78 & 76 & 66 & 58 & - & -- & - & 80 \\
\hline Mo & .51 & .63 & $<10$ & .56 & $<10$ & .61 & $<10$ & .56 & $<0$ & .61 & .51 & .55 & .55 & .55 \\
\hline $\mathrm{Na}$ & - & - & 4.7 & 4.9 & 4.8 & 4.9 & 4.9 & 4.8 & 4.7 & 4.8 & - & -- & -- & -- \\
\hline $\mathrm{Ni}$ & 1.9 & 1.6 & $<10$ & -- & $<10$ & -- & $<10$ & - & $<10$ & -- & 3.4 & 1.9 & 1.7 & -- \\
\hline $\mathrm{Pb}$ & -- & -- & .65 & .49 & $<.5$ & $<.06$ & .64 & .40 & $<.5$ & $<.06$ & -- & -- & -- & $<.06$ \\
\hline $\mathrm{Se}$ & - & - & -- & $<3$ & -. & $<3$ & - & $<3$ & -- & $<3$ & -- & -- & -- & $<3$ \\
\hline $\mathrm{SiO}_{2}$ & -- & - & 6.5 & 7 & 6.3 & 6.5 & 6.8 & 7.2 & 6.3 & 6.5 & 6.4 & 6.7 & 6.8 & -- \\
\hline $\mathrm{Sr}$ & - & -- & 55 & 63 & 55 & 61 & 54 & 63 & 55 & 59 & - & -- & -- & 59 \\
\hline $\mathrm{Tl}$ & -- & -- & -- & $<.05$ & -- & $<.05$ & -- & $<.05$ & -- & $<.05$ & -. & -. & -. & $<.05$ \\
\hline $\mathbf{U}$ & -- & - & -- & $<.06$ & - & $<.06$ & -- & $<.06$ & -- & $<.06$ & -- & -- & -- & .07 \\
\hline $\mathrm{V}$ & 1 & 1.3 & $<6$ & 1.3 & $<6$ & 1 & $<6$ & 1.4 & $<6$ & 1 & .95 & 1.1 & 1.0 & 1 \\
\hline $\mathrm{Zn}$ & .47 & $<10$ & 18 & 10 & $<3$ & .79 & $<3$ & 3.6 & $<3$ & .73 & .46 & .16 & .15 & 1.2 \\
\hline
\end{tabular}


Table 11.-Summary of selected trace-metal concentrations in the Mississippi River below Vicksburg, Mississippi, June 23, 1990

[Samples collected, processed, and analyzed by U.S. Geological Survey National Stream Quality Accounting Network (NASQAN), National Research Program (NRP), Branch of Analytical Services (BAS), and the University of Southern Mississippi (USM). All constituents reported in micrograms per liter, except $\mathrm{Ca}, \mathrm{Mg}, \mathrm{Na}$, and $\mathrm{SiO}_{2}$, which are reported in milligrams per liter. The -- indicate constituents not measured]

\begin{tabular}{|c|c|c|c|c|c|c|c|c|c|c|c|c|c|c|}
\hline \multirow{3}{*}{$\begin{array}{c}\text { Collec- } \\
\text { tion }\end{array}$} & \multicolumn{6}{|c|}{ NASQAN depth-integrated } & \multicolumn{6}{|c|}{ NRP depth-integrated } & \multirow{3}{*}{$\begin{array}{c}\begin{array}{c}\text { USM } \\
\text { grab }\end{array} \\
\text { USM } \\
\text { USM }\end{array}$} & \multirow{3}{*}{$\begin{array}{l}\begin{array}{l}\text { USM } \\
\text { grab }\end{array} \\
\text { NRP } \\
\text { NRP }\end{array}$} \\
\hline & \multirow{2}{*}{$\begin{array}{c}\text { USM } \\
\text { USM }\end{array}$} & \multicolumn{3}{|c|}{ NASQAN } & \multicolumn{2}{|c|}{ NRP } & \multicolumn{2}{|c|}{ NASQAN } & \multicolumn{2}{|c|}{ NRP } & \multirow{2}{*}{$\begin{array}{c}\text { NRP } \\
\text { USM }\end{array}$} & \multirow{2}{*}{$\begin{array}{c}\text { USM } \\
\text { USM }\end{array}$} & & \\
\hline & & USM & BAS & NRP & BAS & NRP & BAS & NRP & BAS & NRP & & & & \\
\hline \multicolumn{15}{|l|}{$\begin{array}{l}\text { Consti- } \\
\text { tuent }\end{array}$} \\
\hline $\mathrm{Ag}$ & - & -- & $<1$ & -- & $<1$ & -- & $<1$ & - & $<1$ & -- & -- & -- & -- & -. \\
\hline Al & 8.7 & 86 & -- & 41 & -- & 120 & -. & 39 & -- & 4.8 & 6.9 & 5.8 & 4.7 & 8.3 \\
\hline As & -- & -- & .- & 2.8 & -. & 2.1 & .- & 2.3 & .- & 1.5 & -- & .- & .. & 2.2 \\
\hline B & - & -- & -- & 52 & -- & 32 & - & 75 & -- & 33 & -- & -- & -- & 37 \\
\hline $\mathrm{Ba}$ & .- & -- & 78 & 72 & 58 & 57 & 78 & 78 & 58 & 56 & .- & -- & -- & 57 \\
\hline $\mathrm{Be}$ & - & - & $<.5$ & .04 & $<.5$ & .03 & $<.5$ & .06 & $<.5$ & $<.02$ & -- & -- & -- & .04 \\
\hline $\mathrm{Ca}$ & - & -. & 37 & 35 & 37 & 36 & 35 & 26 & 37 & 35 & -- & -- & -- & -. \\
\hline $\mathrm{Cd}$ & .05 & - & 1.6 & .80 & $<1$ & $<.1$ & $<1$ & $<.1$ & $<1$ & $<.1$ & $>.2$ & .013 & .014 & $<.1$ \\
\hline Co & -. & -- & $<3$ & .32 & $<3$ & .39 & $<3$ & .30 & $<3$ & .32 & -- & -- & -- & .34 \\
\hline $\mathrm{Cr}_{\mathrm{r}}$ & -- & - & 1.2 & .96 & $<.01$ & .54 & $<.5$ & .68 & $<.5$ & $<.2$ & -. & -- & -- & .12 \\
\hline $\mathrm{Cu}$ & 2.3 & 2.0 & 2.6 & 2.6 & 1.9 & 3.2 & 1.7 & 1.7 & 1.4 & 1.7 & 2.0 & 1.6 & 1.6 & 1.6 \\
\hline $\mathrm{Fe}$ & 1.8 & $>28$ & 30 & - & $<3$ & - & 29 & - & 4.9 & -- & 2.0 & 1.6 & 1.7 & -- \\
\hline $\mathbf{L i}$ & -- & - & 6.7 & 3.2 & 6.7 & 3.1 & 4.9 & 3 & 6.2 & 2.9 & -- & -- & -- & 3 \\
\hline $\mathbf{M g}$ & -- & - & 9.9 & 9.9 & 9.9 & 10 & 9.4 & 7.3 & 9.9 & 9.9 & -- & 10 & 10 & -- \\
\hline Mn & -- & - & 3.8 & 2.9 & $<1$ & 7.7 & 1.5 & .75 & 1.8 & .67 & -- & -- & -. & 2.9 \\
\hline Mo & 1.2 & 1.2 & $<10$ & 1.6 & $<10$ & 1.4 & $<10$ & 1.5 & $<10$ & 1.5 & 1.2 & 1.3 & 1.3 & 1.7 \\
\hline $\mathrm{Na}$ & -. & -- & 10 & 10 & 10 & 11 & 9.9 & 7.7 & 10 & 10 & -- & -- & -- & - \\
\hline $\mathbf{N i}$ & 1.5 & 1.0 & $<10$ & -- & $<10$ & -- & $<10$ & -- & $<10$ & -- & 1.2 & 1.1 & 1.2 & -- \\
\hline $\mathrm{Pb}$ & -- & - & $<.5$ & .08 & $<.5$ & .14 & $<.5$ & .26 & $<.5$ & $<.06$ & -- & -- & -- & $<.06$ \\
\hline $\mathrm{Se}$ & -- & - & -- & $<3$ & - & .16 & -- & $<3$ & - & $<3$ & -. & -- & - & $<3$ \\
\hline $\mathrm{SiO}_{2}$ & - & -- & 6.6 & 6.7 & 6.6 & 7.1 & 6.4 & 5 & 6.6 & 6.7 & 6.8 & 7.0 & 7.0 & -- \\
\hline $\mathrm{Sr}$ & -- & -- & 138 & 155 & 140 & 150 & 135 & 151 & 139 & 152 & -- & -- & -- & 146 \\
\hline $\mathrm{Tl}$ & - & -- & -- & $<.05$ & -- & $<.05$ & -. & $<.05$ & -- & $<.05$ & -- & -. & -- & $<.05$ \\
\hline $\mathbf{U}$ & -- & -- & - & .85 & -- & .83 & -. & .81 & -- & .83 & -- & -- & -- & .66 \\
\hline$v$ & 1.4 & 1.2 & $<6$ & 1.6 & $<6$ & 1.7 & $<6$ & 1.5 & $<6$ & 1.5 & 1.4 & 1.4 & 1.4 & 1.4 \\
\hline $\mathrm{Zn}$ & .32 & 2.1 & $<3$ & 2.3 & $<3$ & 7.1 & $<3$ & 2 & $<3$ & .63 & .31 & .19 & .22 & .79 \\
\hline
\end{tabular}


Table 12.-Summary of selected trace-metal concentrations in the Mississippi River near St. Francisville, Louisiana, June 25, 1990

[Samples collected, processed, and analyzed by U.S. Geological Survey National Stream Quality Accounting Network (NASQAN), National Research Program (NRP), Branch of Analytical Services (BAS), and the University of Southern Mississippi (USM). All constituents reported in micrograms per liter, except $\mathrm{Ca}, \mathrm{Mg}, \mathrm{Na}$, and $\mathrm{SiO}_{2}$, which are reported in milligrams per liter. The -- indicate constituents not measured]

\begin{tabular}{|c|c|c|c|c|c|c|c|c|c|c|c|c|c|c|}
\hline \multirow{3}{*}{$\begin{array}{c}\begin{array}{c}\text { Collec- } \\
\text { tion }\end{array} \\
\text { Pro- } \\
\text { cess } \\
\begin{array}{c}\text { Anal- } \\
\text { ysis }\end{array}\end{array}$} & \multicolumn{6}{|c|}{ NASQAN depth-integrated } & \multicolumn{6}{|c|}{ NRP depth-integrated } & \multirow{3}{*}{$\begin{array}{c}\begin{array}{l}\text { USM } \\
\text { grab }\end{array} \\
\text { USM } \\
\text { USM }\end{array}$} & \multirow{3}{*}{$\begin{array}{c}\begin{array}{l}\text { USM } \\
\text { grab }\end{array} \\
\text { NRP } \\
\text { NRP }\end{array}$} \\
\hline & \multirow{2}{*}{$\frac{\text { USM }}{\text { USM }}$} & \multicolumn{3}{|c|}{ NASQAN } & \multicolumn{2}{|c|}{ NRP } & \multicolumn{2}{|c|}{ NASQAN } & \multicolumn{2}{|c|}{ NRP } & \multirow{2}{*}{$\begin{array}{c}\text { NRP } \\
\text { USM }\end{array}$} & \multirow{2}{*}{$\begin{array}{c}\text { USM } \\
\text { USM }\end{array}$} & & \\
\hline & & USM & BAS & NRP & BAS & NRP & BAS & NRP & BAS & NRP & & & & \\
\hline \multicolumn{15}{|l|}{$\begin{array}{l}\text { Consti- } \\
\text { tuent }\end{array}$} \\
\hline $\mathbf{A g}$ & - & -- & -- & - & $<1$ & $<1$ & -- & $<1$ & -- & -- & -- & -- & - & \\
\hline Al & 2.6 & 18 & - & 17 & - & 13 & - & 22 & - & 13 & - & - & 3.5 & 3.5 \\
\hline As & -- & -- & -- & 2 & -- & 2.1 & -- & 2.2 & -- & 1.9 & -- & -- & - & 1.6 \\
\hline B & -- & -- & - & 33 & -. & 34 & - & 36 & .. & 32 & -. & .. & .- & 32 \\
\hline $\mathbf{B a}$ & -- & - & - & 55 & 56 & 57 & 55 & 56 & 55 & 57 & -- & -. & -. & 57 \\
\hline $\mathrm{Be}$ & -- & - & - & .03 & $<.5$ & $<.02$ & $<.5$ & $<.02$ & $<.5$ & $<.02$ & -- & -- & -- & .04 \\
\hline $\mathrm{Ca}$ & -. & -- & -- & 33 & 35 & 35 & 35 & 35 & 35 & 35 & .. & .. & -. & -. \\
\hline $\mathrm{Cd}$ & .16 & - & -- & $<.1$ & $<1$ & $<.1$ & $<1$ & $<.1$ & $<1$ & $<.1$ & .22 & .017 & .018 & $<.1$ \\
\hline Co & - & - & -- & .29 & $<3$ & .32 & $<3$ & .31 & $<3$ & .30 & -- & -- & -- & .31 \\
\hline $\mathrm{Cr}$ & -- & - & -- & 1.2 & $<.5$ & 21 & 1.7 & 1.2 & 2.1 & $<.2$ & -- & - & -- & $<.2$ \\
\hline $\mathrm{Cu}$ & 2.4 & 3.3 & - & 3.4 & 2.6 & 3.1 & 1.8 & 2.2 & 1.5 & 1.9 & 2.0 & 1.6 & 1.7 & 2 \\
\hline $\mathrm{Fe}$ & 1.8 & 12 & - & - & 3.2 & -- & 12 & -- & 3.1 & - & 1.8 & 1.8 & 1.6 & -. \\
\hline $\mathrm{Li}$ & -- & -- & - & 5.4 & 9.9 & 6 & 7.0 & 2.8 & 6.9 & 2.9 & - & -. & .. & 3.1 \\
\hline $\mathbf{M g}$ & -- & - & -- & 9.3 & 9.5 & 9.9 & 9 & 9.8 & 9.4 & 9.8 & -- & 1 & 1.3 & - \\
\hline $\mathbf{M n}$ & -- & - & - & 2.5 & 2.3 & 3.2 & 1.0 & 1 & 1.2 & 3.1 & - & - & - & 1.5 \\
\hline Mo & 1.5 & 1.7 & - & 1.9 & $<10$ & 1.7 & $<10$ & 1.5 & $<10$ & 1.5 & 1.4 & 1.3 & 1.3 & 1.6 \\
\hline $\mathrm{Na}$ & - & - & - & 10 & 10 & 11 & 9.9 & 10 & 9.9 & 10 & -- & -- & -- &.- \\
\hline $\mathbf{N i}$ & 1.8 & 1.6 & - & -- & $<10$ & - & $<10$ & - & $<10$ & -- & 1.6 & 1.4 & 1.5 & -- \\
\hline $\mathrm{Pb}$ & -- & .- & - & .23 & $<.5$ & $<.06$ & $<.5$ & $<.06$ & $<.5$ & $<.06$ & - & -- & - & $<.06$ \\
\hline $\mathrm{Se}$ & - & - & -- & $<3$ & - & $<3$ & - & $<3$ & - & $<3$ & - & -- & -- & $<3$ \\
\hline $\mathrm{SiO}_{2}$ & -- & -- & -- & 6.4 & 6.5 & 6.7 & 6.4 & 6.8 & 6.5 & 6.7 & 6.9 & 7.0 & 6.9 & -- \\
\hline $\mathrm{Sr}$ & - & -- & - & 146 & 133 & 153 & 130 & 152 & 132 & 152 & - & - & - & 154 \\
\hline $\mathbf{T} \mathbf{1}$ & .. & .. & -- & $<.05$ & - & $<.05$ & -- & $<.05$ & -- & $<.05$ & -- & -- & -- & $<.05$ \\
\hline $\mathbf{U}$ & - & -. & -- & .71 & -- & .73 & - & .78 & -- & .79 & -. & -- & -. & .74 \\
\hline $\mathbf{v}$ & 1.4 & 1.1 & - & 1.5 & $<6$ & 1.6 & $<6$ & 1.5 & $<6$ & 1.5 & 1.6 & 1.5 & 1.5 & 1.5 \\
\hline $\mathrm{Zn}$ & .34 & 3.1 & -- & 4.5 & $<3$ & 1.5 & $<3$ & 2.3 & $<3$ & .93 & .22 & .17 & .18 & .95 \\
\hline
\end{tabular}


Tabie 13.-Summary of seiected trace-metai concentrations in the Mississippi River below Beiie Chasse, Louisiana, June 27, 1990

[Samples collected, processed, and analyzed by U.S. Geological Survey National Stream Quality Accounting Network (NASQAN), National Research Program (NRP), Branch of Analytical Services (BAS), and the University of Southern Mississippi (USM). All constituents reported in micrograms per liter, except $\mathrm{Ca}, \mathrm{Mg}, \mathrm{Na}$, and $\mathrm{SiO}_{2}$, which are reported in milligrams per liter. The -- indicate constituents not measured]

\begin{tabular}{|c|c|c|c|c|c|c|c|c|c|c|c|c|c|c|}
\hline \multirow{3}{*}{$\begin{array}{c}\begin{array}{c}\text { Collec- } \\
\text { tlon }\end{array} \\
\text { Pro- } \\
\text { cess } \\
\begin{array}{c}\text { Anal- } \\
\text { ysls }\end{array}\end{array}$} & \multicolumn{6}{|c|}{ NASQAN depth-Integrated } & \multicolumn{6}{|c|}{ NRP depth-Integrated } & \multirow{3}{*}{$\begin{array}{l}\text { USM } \\
\text { grab }\end{array}$} & \multirow{3}{*}{$\begin{array}{c}\begin{array}{l}\text { USM } \\
\text { grab }\end{array} \\
\text { NRP } \\
\text { NRP }\end{array}$} \\
\hline & \multirow{2}{*}{$\begin{array}{c}\text { USM } \\
\text { USM }\end{array}$} & \multicolumn{3}{|c|}{ NASQAN } & \multicolumn{2}{|c|}{ NRP } & \multicolumn{2}{|c|}{ NASQAN } & \multicolumn{2}{|c|}{ NRP } & \multirow{2}{*}{$\begin{array}{c}\text { NRP } \\
\text { USM }\end{array}$} & \multirow{2}{*}{$\begin{array}{l}\text { USM } \\
\text { USM }\end{array}$} & & \\
\hline & & USM & BAS & NRP & BAS & NRP & BAS & NRP & BAS & NRP & & & & \\
\hline \multicolumn{15}{|l|}{$\begin{array}{l}\text { Consti- } \\
\text { tuent }\end{array}$} \\
\hline Ag & -- & -- & $<1$ & -- & $<1$ & -- & $<1$ & -- & $<1$ & - & -- & -- & -. & -- \\
\hline Al & 8.7 & 22 & - & 17 & -- & 87 & -- & 16 & -- & 5.6 & 9.2 & 6.7 & 7.7 & 4.1 \\
\hline As & -. & -- & -- & 2.1 & .- & 2.2 & -- & 2.4 & .- & 1.7 & -- & -- & -- & 2.2 \\
\hline B & -. & -- & - & 34 & -- & 33 & -- & 35 & -- & 26 & -- & -- & -- & 35 \\
\hline $\mathrm{Ba}$ & -- & -- & 59 & 57 & 59 & 58 & 58 & 56 & 59 & 58 & .. & -- & -- & 62 \\
\hline $\mathrm{Be}$ & - & $<1$ & $>.5$ & .02 & $<.5$ & $<.02$ & $<.5$ & $<.02$ & $<.5$ & $<.02$ & - &.- & -- & $<.02$ \\
\hline $\mathrm{Ca}$ & - & -- & 36 & 35 & 37 & 35 & 36 & 35 & 37 & 35 & -- & .- & .- & .. \\
\hline $\mathrm{Cd}$ & .09 & -- & $<1$ & $<.1$ & $<1$ & $<.1$ & $<1$ & $<.1$ & $<1$ & $<.1$ & $>.2$ & .022 & .02 & $<.1$ \\
\hline Co & -- & -. & $<3$ & .32 & $<3$ & .33 & $<3$ & .30 & $<3$ & .24 & -- & -- & -. & .30 \\
\hline $\mathrm{Cr}$ & -- & -- & $<.5$ & $<.2$ & $<.5$ & .25 & $>.5$ & .24 & $<.5$ & $<.2$ & -- & -- & - & $<.2$ \\
\hline $\mathrm{Cu}$ & -- & 3.8 & 3.7 & 4.2 & 3.3 & 3.8 & 1.7 & 2.5 & 1.4 & 1.6 & 24 & 1.8 & 1.6 & 1.6 \\
\hline $\mathrm{Fe}$ & 1.8 & 10 & 7.7 & - & 3.4 & -- & 5.9 & -- & 7.6 & -- & 1.5 & 1.4 & 1.5 & -- \\
\hline $\mathbf{L i}$ & -- & -- & 7.1 & 3.3 & 7.4 & 3.3 & 6.7 & 3.1 & 6.4 & 2.8 & -. & .. & -. & 3.3 \\
\hline $\mathbf{M g}$ & - & - & 9.8 & 9.8 & 9.9 & 9.8 & 9.8 & 9.9 & 9.9 & 9.9 & -- & 10 & 10 & -- \\
\hline Mn & -- & -- & 1.5 & 1.7 & $<1$ & 4.0 & $<1$ & .83 & 4.3 & .76 & -- & - & .. & 1.2 \\
\hline Mo & 1.5 & 1.4 & $<10$ & 1.6 & $>10$ & 1.6 & $<10$ & 1.6 & $<10$ & 1.5 & 1.7 & 1.4 & 1.2 & 1.7 \\
\hline $\mathrm{Na}$ & -- & -- & 12 & 12 & 12 & 12 & 12 & 12 & 12 & 12 & -- & .- & -- & -- \\
\hline $\mathrm{Ni}$ & 1.9 & 1.4 & $<10$ & - & $<10$ & - & $<10$ & - & $<10$ & -- & 1.7 & 1.6 & 1.5 & -- \\
\hline $\mathrm{Pb}$ & -- & -. & $<.5$ & $<.06$ & 1.0 & .13 & $<.5$ & .19 & $<.5$ & $<.06$ & -- & -- & -- & $<.06$ \\
\hline $\mathrm{Se}$ & -- & - & -- & $<3$ & -- & $<3$ & -- & $<3$ & -- & $<3$ & -- & -- & -- & $<3$ \\
\hline $\mathrm{SiO}_{2}$ & -- & -- & 6.7 & 6.8 & 6.8 & 7.0 & 6.7 & 6.8 & 6.8 & 6.8 & 7.0 & 7.1 & 7.0 & -- \\
\hline $\mathbf{S r}$ & -- & -- & 138 & 153 & 143 & 155 & 141 & 158 & 141 & 154 & - & -. & -- & 152 \\
\hline $\mathrm{Tl}$ & -- & -- & -- & $<.05$ & .. & $<.05$ & -- & $<.05$ & -- & $<.05$ & -- & -- & -- & $<.05$ \\
\hline $\mathrm{U}$ & -. & -. & - & .74 & -- & .75 & -- & .79 & -- & .64 & - & -- & -- & .60 \\
\hline V & 1.6 & 1.4 & $<6$ & 1.7 & $<6$ & 1.9 & $<6$ & 1.9 & $<6$ & 1.5 & 1.9 & 1.9 & 1.8 & 1.9 \\
\hline $\mathrm{Zn}$ & .41 & 5.5 & 3.4 & 4.2 & $<3$ & 2.4 & $<3$ & 4.1 & $<3$ & 1.2 & .18 & .3 & .19 & 1.3 \\
\hline
\end{tabular}




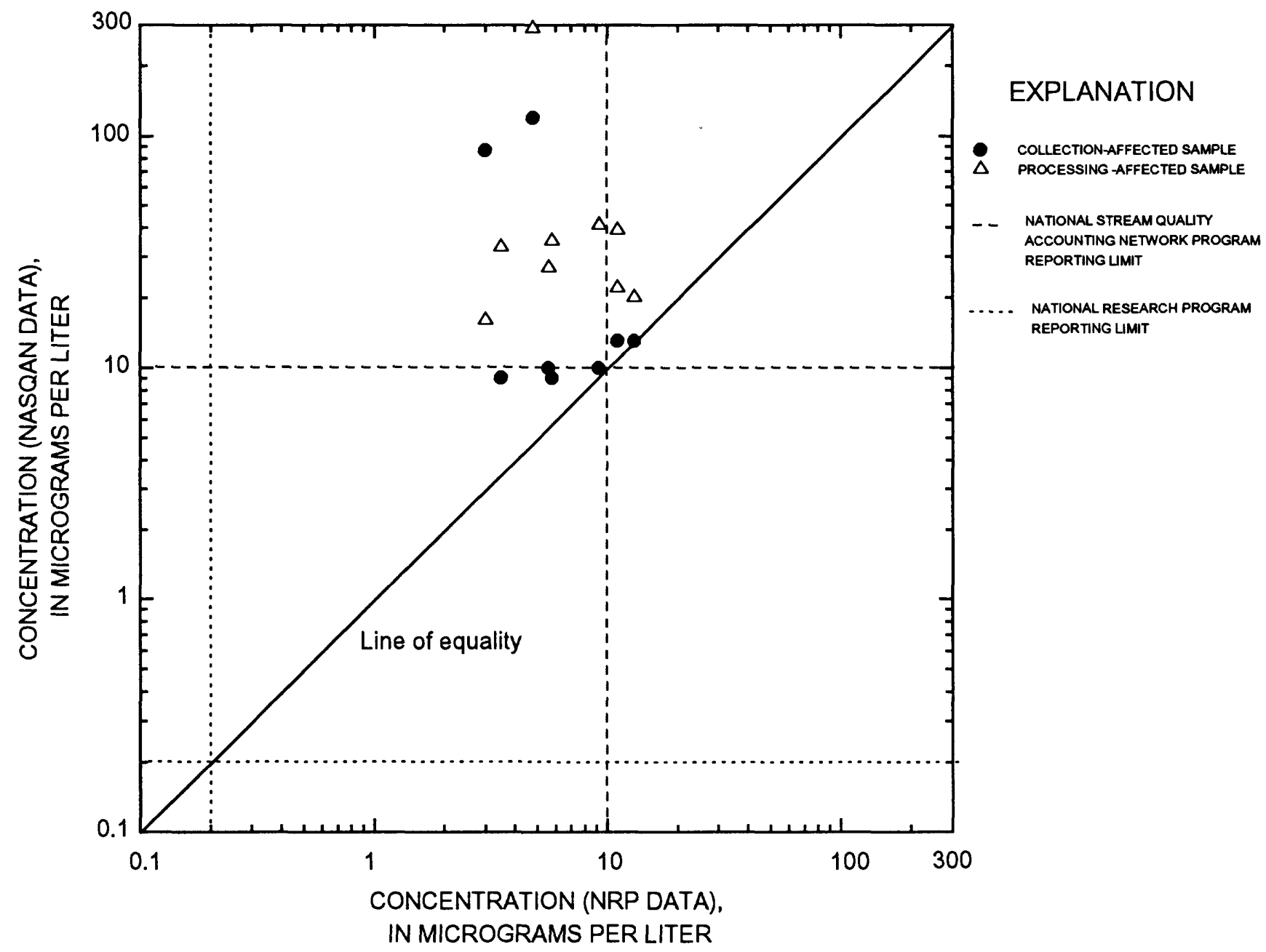

Figure 4. Relation between dissolved aluminum concentrations in samples collected and processed by U.S. Geological Survey National Stream Quality Accounting Network Program (NASQAN) and National Research Program (NRP), June 1990. 


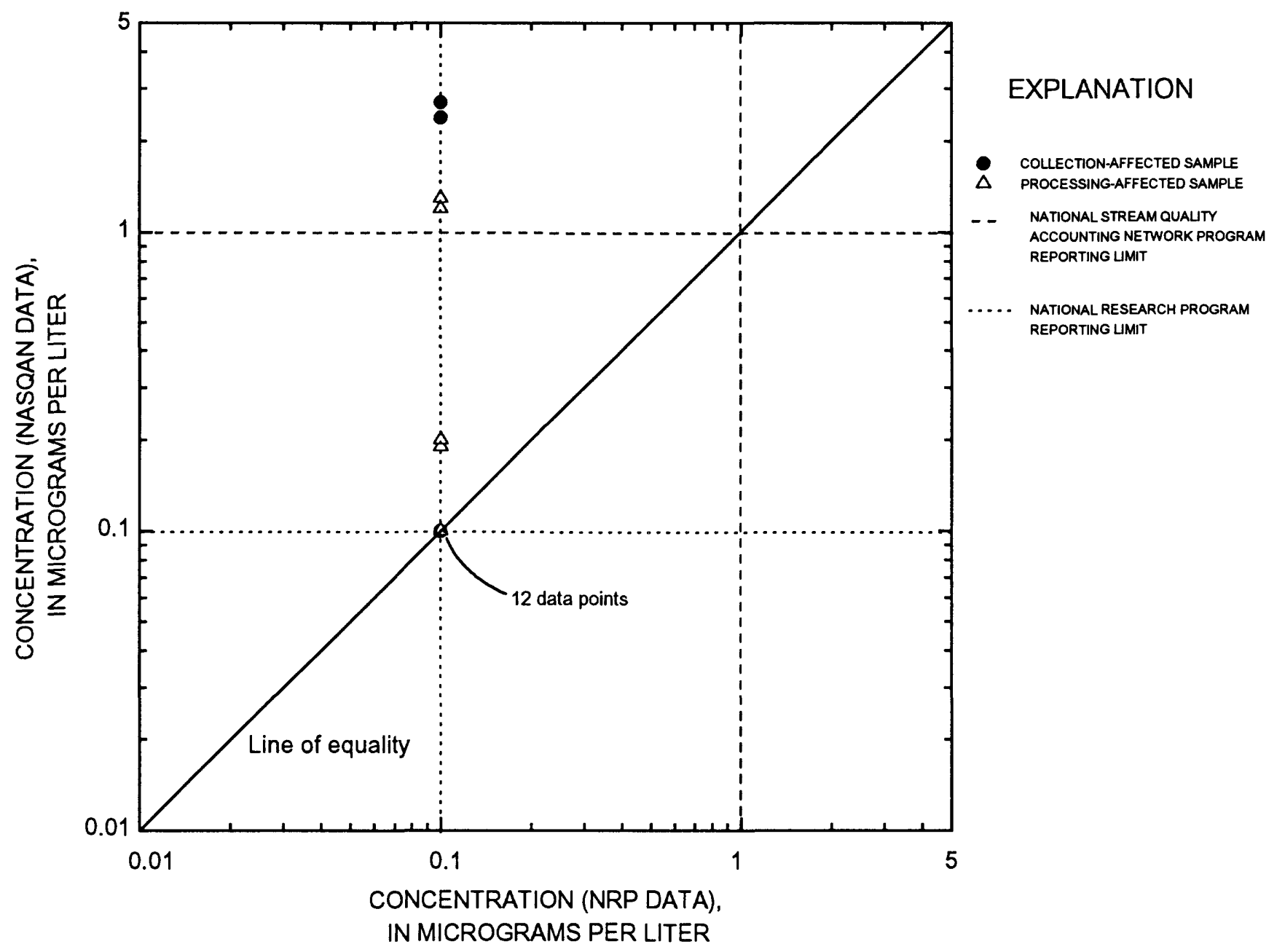

Figure 5. Relation between dissolved cadmium concentrations in samples collected and processed by U.S. Geological Survey National Stream Quality Accounting Network Program (NASQAN) and National Research Program (NRP), June 1990. 


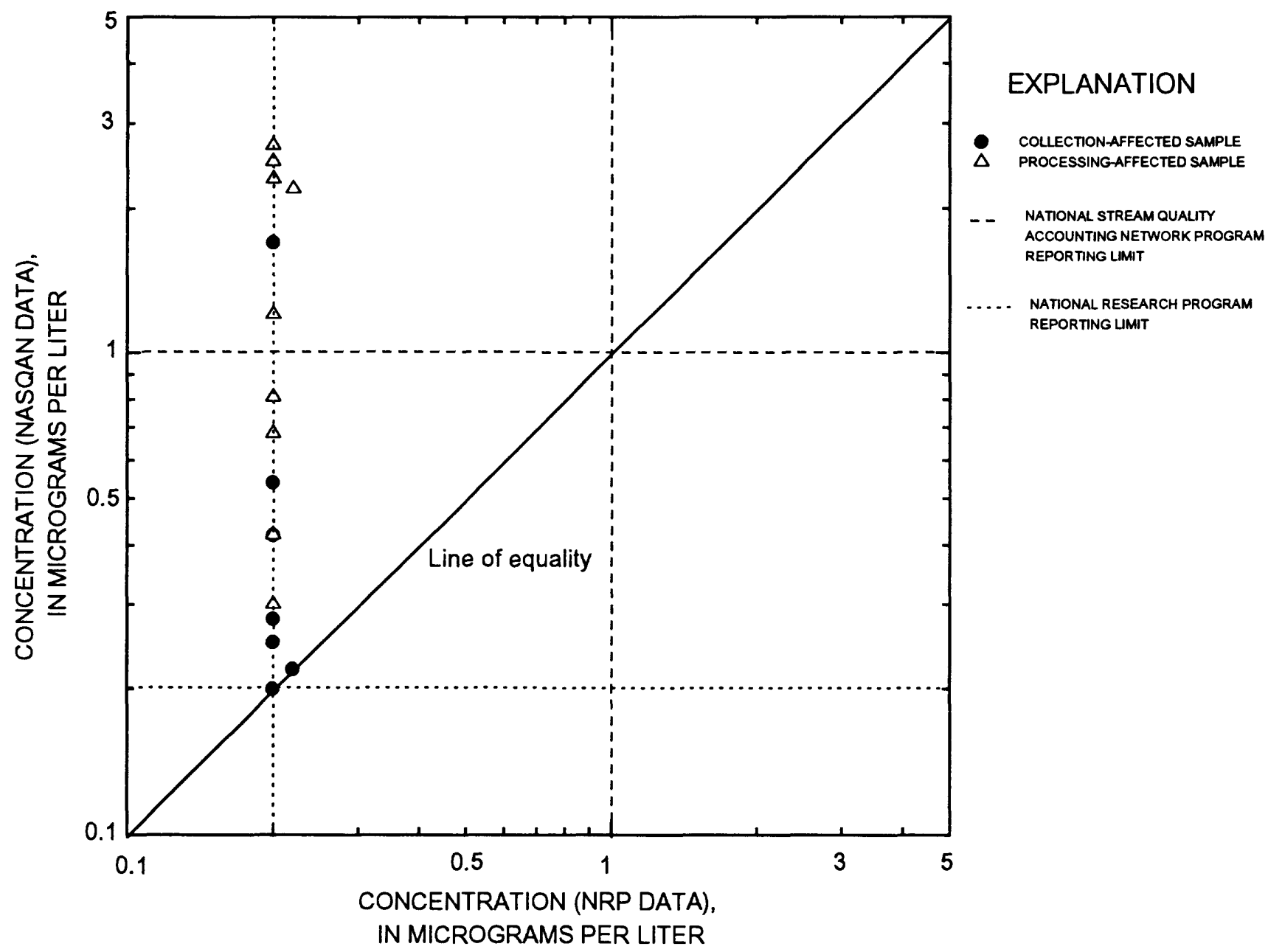

Figure 6. Relation between dissolved chromium concentrations in samples collected and processed by U.S. Geological Survey National Stream Quality Accounting Network Program (NASQAN) and National Research Program (NRP), June 1990. 


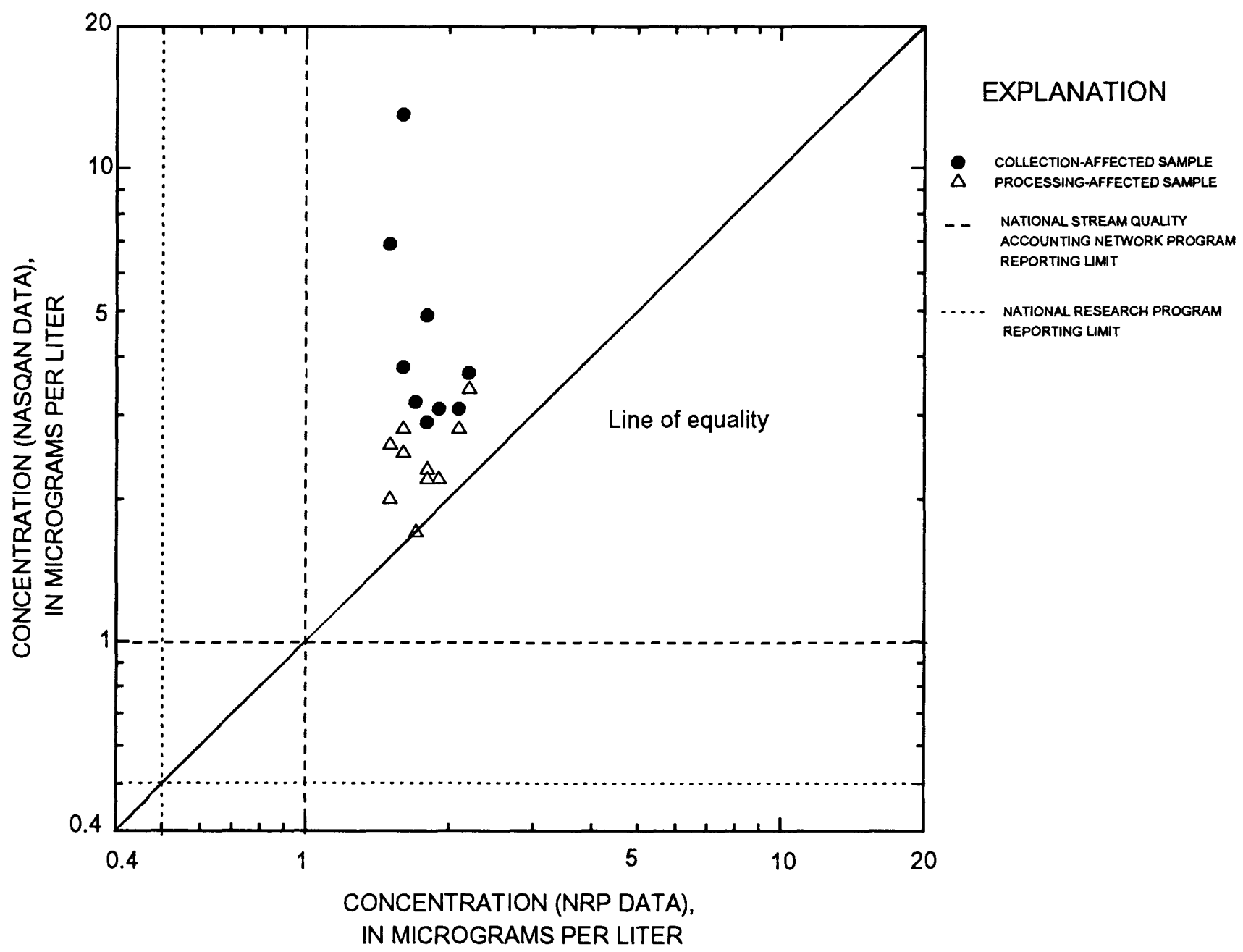

Figure 7. Relation between dissolved copper concentrations in samples collected and processed by U.S. Geological Survey National Stream Quality Accounting Network Program (NASQAN) and National Research Program (NRP), June 1990. 


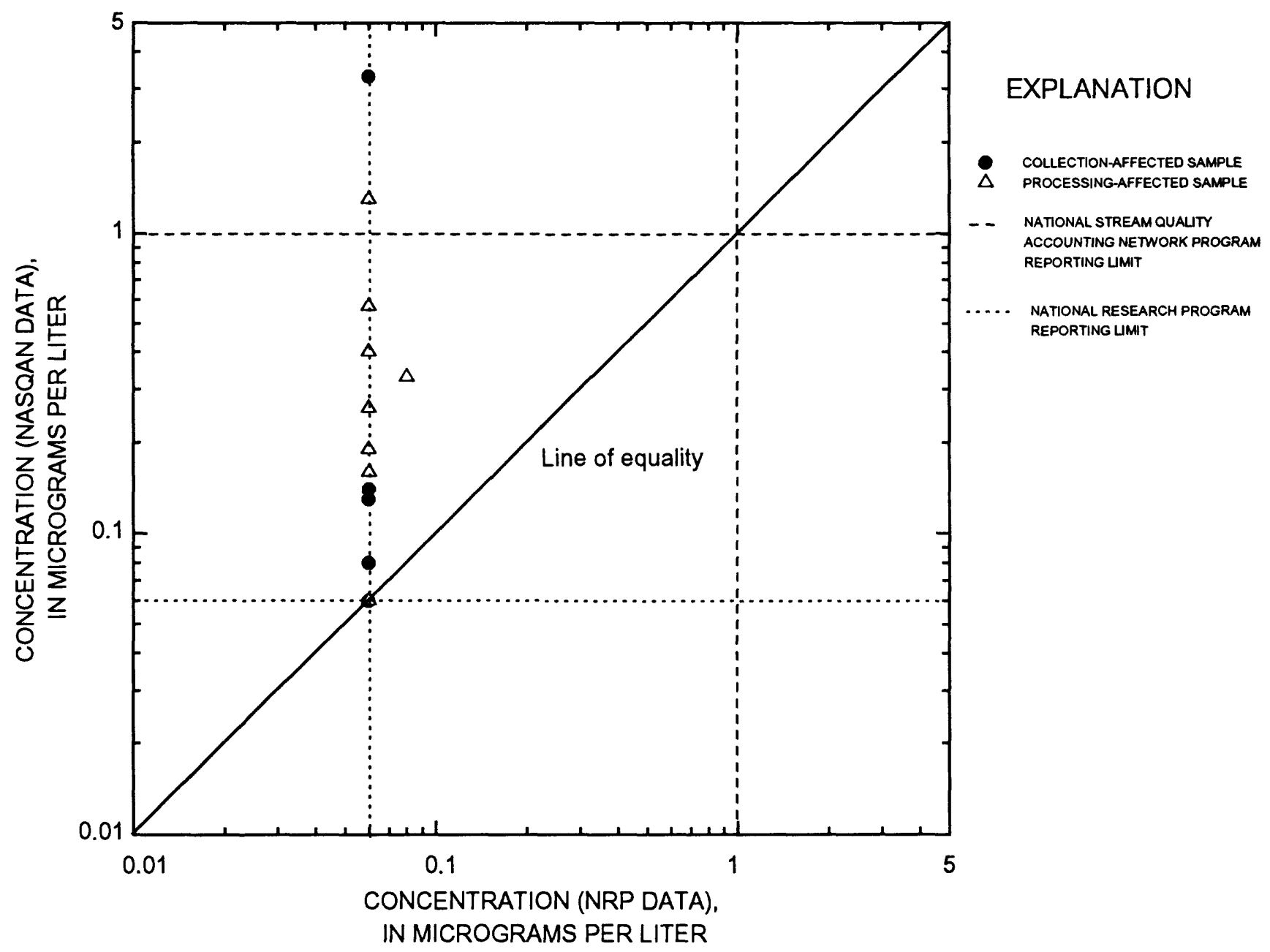

Figure 8. Relation between dissolved lead concentrations in samples collected and processed by U.S. Geological Survey National Stream Quality Accounting Network Program (NASQAN) and National Research Program (NRP), June 1990. 


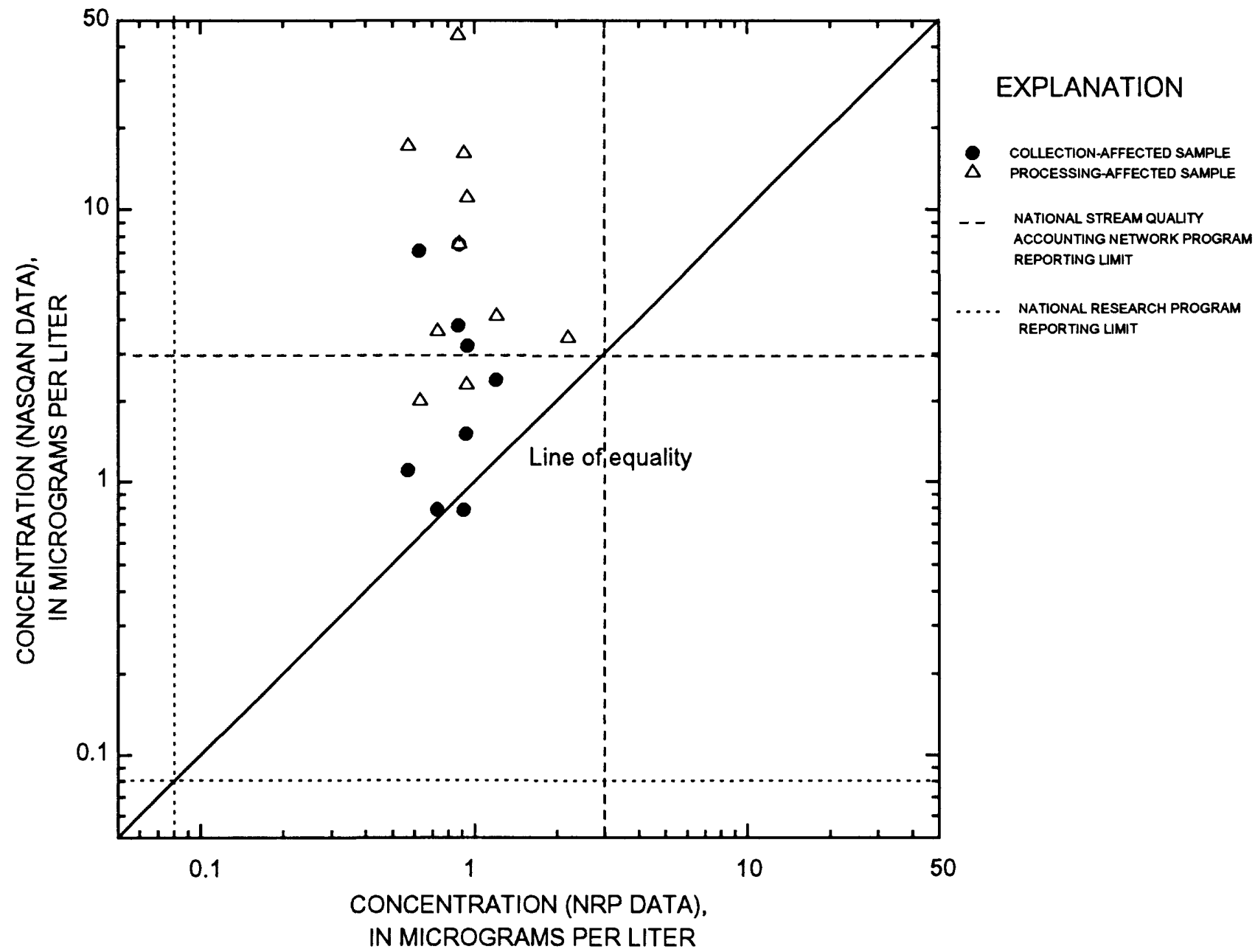

Figure 9. Relation between dissolved zinc concentrations in samples collected and processed by U.S. Geological Survey National Stream Quality Accounting Network Program (NASQAN) and National Research Program (NRP), June 1990. 


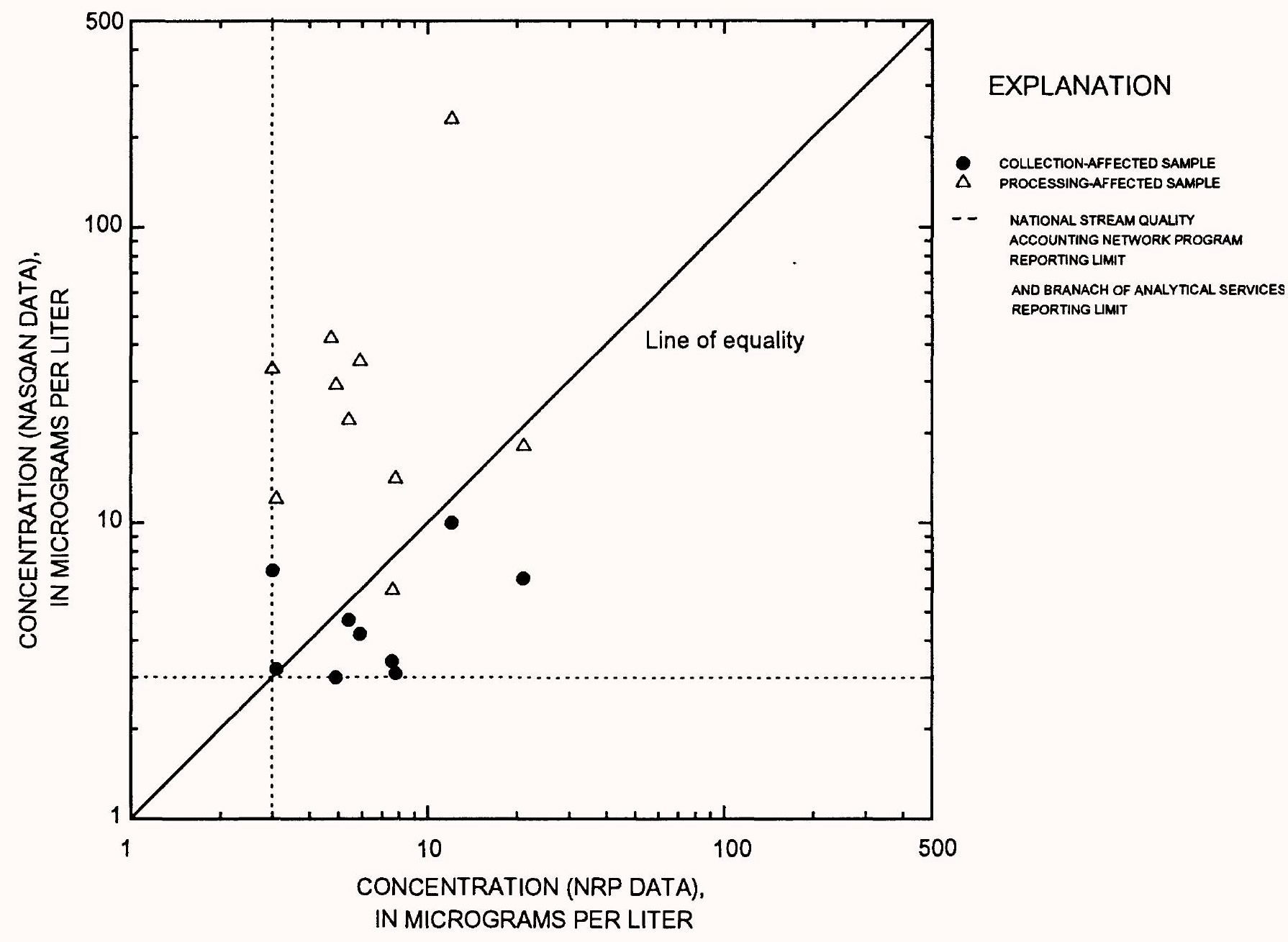

Figure 10. Relation between dissolved iron concentrations in samples collected and processed by U.S. Geological Survey National Stream Quality Accounting Network Program (NASQAN) and National Research Program (NRP) (analysis by Branch of Analytical Services), June 1990. 


\section{REFERENCES CITED}

Boyle, E.A., Huested, S.S., and Jones, S.P., 1981, On the distribution of copper, nickel, and cadmium in the surface waters of the North Atlantic and North Pacific Ocean: Journal of Geophysical Research, v. 86, p. 8048-8066.

1982, The chemical mass balance of the Amazon Plume-Copper, nickel and cadmium : Deep-Sea Research, v. 29, p. 1355-1364.

Briggs, J.C., and Ficke, J.F., 1978, Quality of rivers of the United States, 1975 water year: U.S. Geological Survey Open-File Report 78-200, 436 p.

Garbarino, J.R., and Taylor, H.E., 1979, An inductively-coupled plasma atomic-emission spectrometric method for routine water quality testing: Applied Spectroscopy, v. 33, no. 3, p. 220-226.

1980, A babington-type nebulizer for use in the analysis of natural water samples by inductively coupled plasma spectrometry, Applied Spectroscopy: v. 34, no. 3 , p. 393-399.

Kuehner, E.C., Alvarez, R., Paulsen, P.J., and Murphy, T.J., 1972, Production and analysis of special high-purity acids purified by sub-boiling distillation: Analytical Chemistry, v. 44 , no. 12 , p. 2050.

Leenheer, J.A., Meade, R.H., Taylor, H.E., and Pereira, W.E., 1989, Sampling, fractionation, and dewatering of suspended sediment from the Mississippi River for geochemical and trace-contaminant analysis, in Mallard, G.E., and, Ragone, S.E., eds., U.S. Geological Survey Toxic Substances Hydrology Program-Proceedings of the Technical Meeting, Phoenix, Arizona, Sept. 26-30, 1988: U.S. Geological Survey Water-Resources Investigations Report 88-4220, p. 501-511.

Meade, R.H., 1985, Suspended sediment in the Amazon River and its tributaries in Brazil during 1982-84: U.S. Geological Survey Open-File Report 85-492, 34 p.

Meade, R.H., Dunne, T., Richey, J.E., Santos, U. de M., and Salati, E., 1985. Storage and remobilization of suspended sediment in the lower Amazon River of Brazil: Science, v. 228, p. $488-490$.

Meade, R.H., and Stevens., H.H., Jr., 1990, Strategies and equipment for sampling suspended sediment and associated toxic chemicals in large rivers-with emphasis on the Mississippi River: The Science of the Total Environment, v. 97/98, p. 125-136.

Moody, J.A., and Meade, R.H., 1992, Hydrologic and sedimentologic data collected during three cruises at low water on the Mississippi River and some of its tributaries, July 1987-June 1988: U.S. Geological Survey Open-File Report 91-485, 143 p.

1993, Hydrologic and sedimentologic data collected during four cruises at high water on the Mississippi River and some of its tributaries, March 1989-June 1990:

U.S. Geological Survey Open-File Report 92-651, 227 p. 
Nordin, C.F., Jr., Cranston, C.C., and Mejia, B.A., 1983, New technology for measuring water and suspended-sediment discharge of large rivers, in Proceedings of the International Symposium on River Sedimentation, 2d, Nanjing, China, Water Resources and Electric Power Press, p. 1145-1158.

Pereira, W.E., Rostad, C.E., and Leiker, T.J., 1990, Distribution of agrochemicals in the lower Mississippi River and its tributaries: The Science of the Total Environment, v. 97/98, p. 41-53.

Shiller, A.M., and Boyle, E., 1983, Trace metals in the plume of the Mississippi River: Eos, v. 64, p. 1021.

1987, Variability of dissolved trace metals in the Mississippi River: Geochimica et Cosmochimica Acta, v. 51, p. 3273-3277.

Skougstad, M.W., Fishman, M.J., Friedman, L.C., Erdmann, D.E., and Duncan, S.S., 1979, Methods for the determination of inorganic substances in water and fluvial sediments: U.S. Geological Survey Techniques of Water-Resource Investigations, book 5, chap. A1, p. 229.

Smith, R.A., Alexander, R.B., and Wolman, M.G., 1987, Water-quality trends in the Nation's rivers: Science, v. 235, p. 1607-1615.

Taylor, H.E., 1989, Hydrologic applications of ICP-MS, in Date, A., and Gray, A.L., eds., Applications of ICPMS: London, Blackie, p. 71-89.

Taylor, H.E., Garbarino, J.R., and Brinton, T.I., 1990, The occurrence and distribution of trace metals in the Mississippi River and its tributaries: The Science of the Total Environment, v. 97/98, p. 369-384.

Taylor, H.E., and Garbarino, J.R., 1991, The measurement of trace metals in water resource monitoring samples by inductively coupled plasma-mass spectrometry: Spectrochimica Acta Reviews, v. 14, p. 33-44.

Windom, H.L., Byrd, J.T., Smith, R.G., Jr., and Haun, F., 1991, Inadequacy of NASQAN data for assessing metal trends in the Nation's rivers: Environmental Science and Technology: v. 25, p. 1137-1142. 